\title{
Osteoclast-specific cathepsin K deletion stimulates S1P-dependent bone formation
}

\author{
Sutada Lotinun, ${ }^{1}$ Riku Kiviranta, ${ }^{1,2}$ Takuma Matsubara, ${ }^{1}$ Jorge A. Alzate, ${ }^{1}$ Lynn Neff, ${ }^{1}$ Anja Lüth, ${ }^{3}$ \\ Ilpo Koskivirta, ${ }^{2}$ Burkhard Kleuser, ${ }^{3}$ Jean Vacher, ${ }^{4}$ Eero Vuorio, ${ }^{2}$ \\ William C. Horne, ${ }^{1}$ and Roland Baron ${ }^{1,5}$
}

\begin{abstract}
1'Department of Oral Medicine, Infection and Immunity, Harvard School of Dental Medicine, Boston, Massachusetts, USA.
2Departments of Medical Biochemistry and Genetics and Medicine, University of Turku, Turku, Finland. ${ }^{3}$ University of Potsdam, Institute of Nutritional Science, Department of Nutritional Toxicology, Nuthetal (Bergholz-Rehbrücke), Germany. ${ }^{4}$ Clinical Research Institute of Montreal, Faculté de Médecine de l'Université de Montréal, Montreal, Quebec, Canada. ${ }^{5}$ Harvard Medical School, Endocrine Unit, Massachusetts General Hospital, Boston, Massachusetts, USA.
\end{abstract}

\begin{abstract}
Cathepsin K (CTSK) is secreted by osteoclasts to degrade collagen and other matrix proteins during bone resorption. Global deletion of Ctsk in mice decreases bone resorption, leading to osteopetrosis, but also increases the bone formation rate (BFR). To understand how Ctsk deletion increases the BFR, we generated osteoclast- and osteoblast-targeted Ctsk knockout mice using floxed Ctsk alleles. Targeted ablation of Ctsk in hematopoietic cells, or specifically in osteoclasts and cells of the monocyte-osteoclast lineage, resulted in increased bone volume and BFR as well as osteoclast and osteoblast numbers. In contrast, targeted deletion of Ctsk in osteoblasts had no effect on bone resorption or BFR, demonstrating that the increased BFR is osteoclast dependent. Deletion of Ctsk in osteoclasts increased their sphingosine kinase 1 (Sphk1) expression. Conditioned media from Ctsk-deficient osteoclasts, which contained elevated levels of sphingosine-1-phosphate $(\mathrm{S} 1 \mathrm{P})$, increased alkaline phosphatase and mineralized nodules in osteoblast cultures. An $S_{1} \mathrm{P}_{1,3}$ receptor antagonist inhibited these responses. Osteoblasts derived from mice with Ctsk-deficient osteoclasts had an increased $R A N K L / O P G$ ratio, providing a positive feedback loop that increased the number of osteoclasts. Our data provide genetic evidence that deletion of CTSK in osteoclasts enhances bone formation in vivo by increasing the generation of osteoclast-derived S1P.
\end{abstract}

\section{Introduction}

One of the major issues with the use of currently available antiresorptive drugs for the treatment of osteoporosis is that while they are very efficient at reducing osteoclast numbers and activity, leading to markedly reduced bone resorption, they also profoundly reduce bone formation. The reduction in activation frequency of bone remodeling units along bone surfaces and the coupling between bone resorption and formation result in a decrease in bone formation following treatment (1). Furthermore, it has been argued that such profound inhibition of bone turnover could be contributing to rare, but significant, clinical complications such as osteonecrosis of the jaw (ONJ) and atypical subtrochanteric femoral fractures $(2-5)$. The profound decrease in bone remodeling associated with these drugs has also been suggested to decrease the responsiveness of the skeleton to parathyroid hormone $(\mathrm{PTH})$, the only anabolic drug currently available in the clinic (6). In this context, it became important to identify novel therapeutic pathways through which bone resorption could be inhibited efficiently while maintaining bone turnover and bone formation at clinically acceptable levels.

Information about what these novel therapeutic approaches could actually accomplish came from the identification of several genes whose mutation or deletion caused osteopetrosis, a phenotype in which defects in osteoclasts lead to reduced bone resorption and high bone density. Careful analysis of the changes in

Authorship note: Sutada Lotinun and Riku Kiviranta contributed equally to this work. Conflict of interest: Roland Baron is a consultant for Merck and receives research support from this company.

Citation for this article: J Clin Invest. 2013;123(2):666-681. doi:10.1172/JCI64840. bone remodeling activity in these human and/or murine mutants showed that, as with currently available antiresorptive drugs, bone formation was always decreased in mutations leading to deficient osteoclast differentiation and numbers, such as those affecting RANK or RANKL $(7,8)$. In contrast, bone formation was maintained or even increased in mutations leading to strictly functional defects in osteoclasts, such as mutations of the chloride channel CLC-7, VATPase subunits, or carbonic anhydrase (9). One such mutation affects cathepsin $\mathrm{K}$, a cysteine protease secreted by osteoclasts that is essential for the degradation of matrix collagen and the activation of tartrate-resistant acid phosphatase (TRAP) $(10,11)$.

In humans, mutation of cathepsin $\mathrm{K}$ leads to pycnodysostosis, a rare autosomal recessive skeletal dysplasia in which osteoclast function is defective. It is characterized by short stature, osteopetrosis, acroosteolysis (resorption of distal phalanges), spondylolysis, delayed cranial suture closure, and bone fragility (12-14). Several mouse models of germline cathepsin $\mathrm{K}$ deficiency have also been generated by deleting Ctsk, the gene encoding cathepsin $\mathrm{K}$ (15-19). These animal models showed reduced bone resorption, together with normal or increased bone formation, contributing to the osteopetrotic phenotype. These studies therefore confirmed that it is possible to decrease bone resorption without the inevitable reduction in bone formation. Mechanistically, however, Ctsk was globally deleted in all these studies, making it impossible to determine whether the increased bone formation resulted from cell-autonomous effects in cells other than osteoclasts, including cells of the osteoblast lineage, or was secondary to changes in osteoclast-produced osteoanabolic coupling factors.

To address this important mechanistic question, we generated mice in which the Ctsk allele was specifically deleted in cells 
A

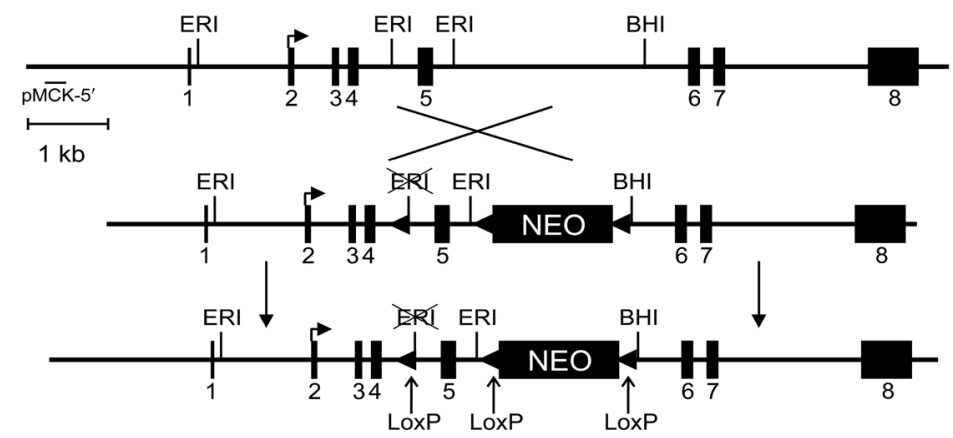

Cre recombinase activity $\longrightarrow$

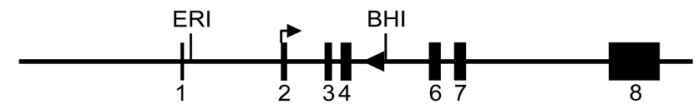

B

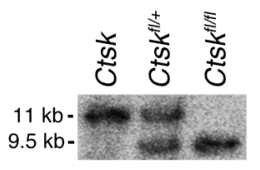

C

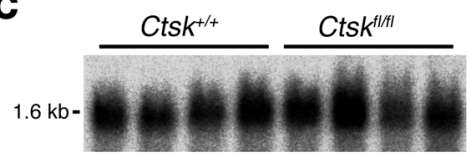

\section{Figure 1}

Generation of $\mathrm{Ctsk}^{\mathrm{fl} / \mathrm{fl}}$ mouse line. (A) To generate the targeting construct pG3NEOBLoxP-2, the expression cassette for the neomycin resistance gene (NEO) flanked by loxP sites (large arrowheads) was inserted in reverse orientation between the EcoRI (ERI) and the BamHI (BHI) sites in intron 5 of the Ctsk gene. Successful targeting of the Ctsk locus resulted in insertion of the 3 loxP sites and the NEO gene into the Ctsk gene. Cre recombinase activity resulted in sequential removal of the sequences between the loxP sites including exon 5 and the NEO cassette, leaving only one loxP site in the locus. (B) The results of the Southern hybridization of tail DNA from control, Ctsk ${ }^{f l /}$, and Ctsk ${ }^{f l / f l}$ mice indicating a band of $11 \mathrm{~kb}$ for the wild-type allele, and $9.5 \mathrm{~kb}$ for the mutant allele after a digestion with $\mathrm{BHI}$ restriction enzyme and hybridization with probe pMCK- $5^{\prime}$. (C) Northern analysis of humerus total RNA demonstrating normal Ctsk mRNA expression in $\mathrm{Ctsk}^{\mathrm{fl} / \mathrm{fl}}$ mice. (D) $\mathrm{pQCT}$ analysis shows normal femoral BMD in Ctsk ${ }^{f / f l}$ mice.
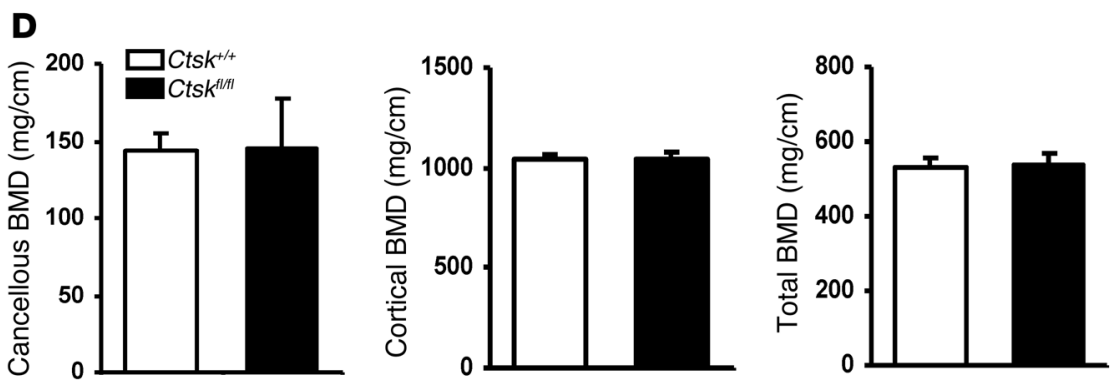

of the hematopoietic lineage, in the osteoclast lineage, or in the osteoblast lineage using the Cre-loxP system, and analyzed their skeletal phenotypes. We determined the effects of these cell-specific deletions on bone resorption and bone formation, and characterized the molecular mechanism underlying these changes. Targeted deletion of Ctsk in hematopoietic cells, or more specifically, in the osteoclast lineage, resulted in mild osteopetrosis with an increase in the number of poorly functional osteoclasts, as well as an increase in osteoblasts and bone formation. In vitro assays of primary osteoblasts derived from long bones of these conditional knockout mice showed an increase in osteoprogenitors, alkaline phosphatase (ALP), and mineralized bone nodules. In contrast, ablation of Ctsk in cells of the osteoblast lineage had no effect on either bone formation or bone resorption, suggesting that the increased bone formation seen in the mice with germline deletion of Ctsk results from increased coupling between osteoclasts and osteoblasts. A search for molecules that could mediate this increase in the coupling mechanism showed a significant increase in sphingosine kinase 1 (Sphk1) in Ctsk-deleted osteoclasts. Sphk1 catalyzes the phosphorylation of sphingosine to sphingosine-1phosphate (S1P), which promotes osteoblast differentiation and bone-forming activity (20). Consistent with the increased Sphk1, conditioned medium derived from Ctsk-deleted osteoclasts contained more S1P and ALP in vitro. Furthermore, the RANKL/OPG ratio was increased in osteoblasts derived from long bones of these mice, suggesting a positive feedback loop to increase the number of osteoclasts in the absence of cathepsin $\mathrm{K}$. These results demon- strate that the increased bone formation rate (BFR) observed after deletion of Ctsk is osteoclast mediated, and establish the fact that osteoclasts can indeed promote bone formation in an in vivo setting. Furthermore, these findings provide additional evidence that $\mathrm{S} 1 \mathrm{P}$ is an important osteoclast-derived anabolic factor, a clastokine, that couples bone resorption to bone formation.

\section{Results}

Generation of a cathepsin K conditional knockout mouse. We generated mice carrying the floxed Ctsk allele by inserting loxP sites flanking exon 5 that encodes for one of the active site cysteine residues of cathepsin $\mathrm{K}\left(\mathrm{Ctsk}^{f l}\right)$ (Figure 1A). The point mutations in this area are sufficient to cause pycnodysostosis disease in humans. Southern analysis showed a wild-type band of $11 \mathrm{~kb}$ and a mutant band of $9.5 \mathrm{~kb}$ (Figure 1B). Normal Ctsk mRNA expression was observed in Ctskflfl mice using Northern analysis (Figure 1C). To ensure that insertion of the loxP sites did not affect bone mass, we examined the long bones of the Ctskflfl mice by peripheral quantitative CT (pQCT). The analysis showed that cancellous, cortical, and total bone mineral density (BMD) in Ctsklffl mice was similar to that in $\mathrm{Ctsk}^{+/+}$mice (Figure 1D).

Hematopoietic cell-and osteoclast-specific deletion of Ctsk. To examine the role of cell-specific targeted deletion of Ctsk in bone remodeling, we first determined the effects of Ctsk deletion in hematopoietic cells, including osteoclasts, using the Mx1-Cre mice (21), or more specifically, in the monocyte-osteoclast lineage using the CD11b-Cre mice (22-24). Ctskfl/fl mice were crossed with those expressing Cre 
A

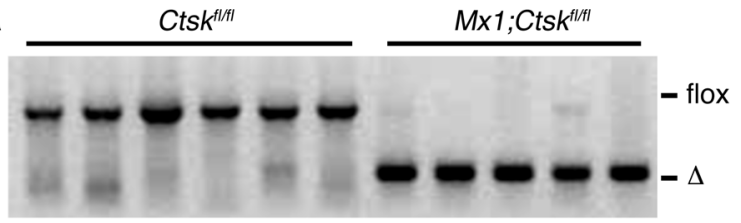

B

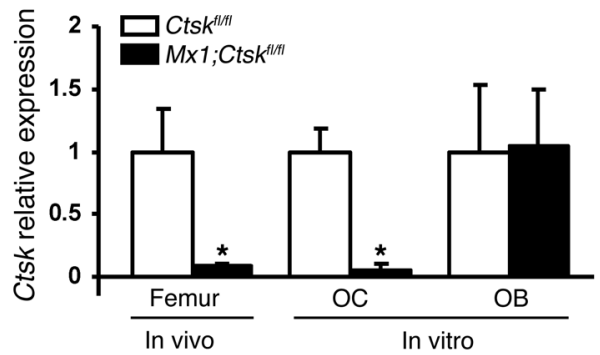

C

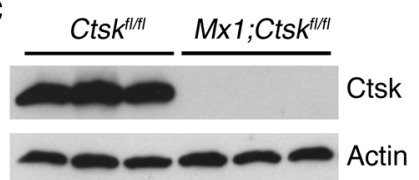

D

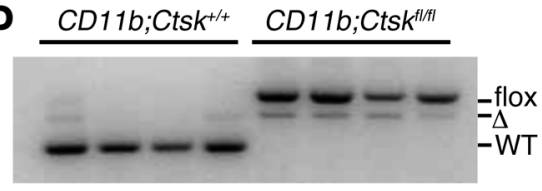

E

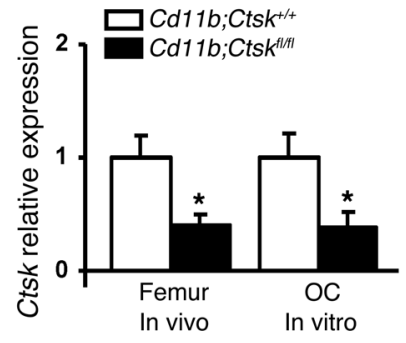

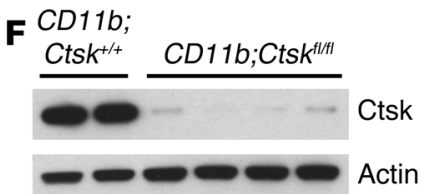

Figure 2

Ablation of Ctsk in hematopoietic cells $\left(M \times 1 ; C t s k^{f l f f}\right)$ and osteoclasts (CD11b;Ctsk ${ }^{f / / 1}$ ) suppresses Ctsk expression. (A and D) PCR of genomic DNA from spleen showed the deletion of Ctsk exon $(\Delta)$. (B and E) qRT-PCR analysis of Ctsk showed a decrease in Ctsk mRNA expression in bone and osteoclasts (OC), but not osteoblasts (OB). (C and F) Western blot analysis of cathepsin $\mathrm{K}$ in protein lysates from osteoclasts derived from $M \times 1 ; C t s k^{f l / f l}$ or CD11b;Ctsk ${ }^{f l f l}$ mice and controls. The gels in A, C, D, and $F$ show samples from multiple animals per condition. Results are mean \pm SEM. ${ }^{*} P<0.05$ versus controls. recombinase under the control of a type I interferon-inducible $\mathrm{Mx} 1$

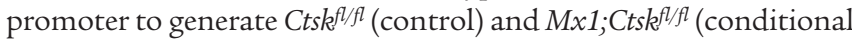
knockout) mice and those expressing Cre recombinase under the control of CD11b promoter to generate CD11b; $\mathrm{Ctsk}^{+/+}$(control) and

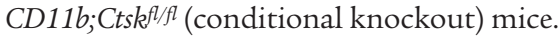

Induction of $M x 1$-Cre expression by intraperitoneal injection of polyinosinic-polycytidylic acid (polyI:C) at 2 weeks of age led to the complete deletion of the Ctsk exon 5 in spleen DNA (Figure 2A), reduced Ctsk mRNA levels in femur and osteoclasts by $92 \%$ and $94 \%$, respectively (Figure 2B), and markedly diminished cathepsin K protein levels at 9 weeks of age (Figure 2C). It was previously suggested that the Cre recombinase in $M x 1$-Cre transgenic mice is expressed not only in osteoclasts, but also in osteoblasts (25). However, quantitative real-time PCR (qRT-PCR) analysis of the Mx1;Ctskl/fl mice indicated that the Ctsk gene, which is expressed at much lower levels $(0.5 \%)$ than in osteoclasts, was not deleted in osteoblasts (Figure $2 \mathrm{~B}$ ). $\mathrm{CD} 11 \mathrm{~b}$-driven Cre recombinase expression resulted in deletion of exon 5 in spleen genomic DNA (Figure 2D). Western blotting analysis showed a reduction of more than $90 \%$ in cathepsin $\mathrm{K}$ protein levels in CD11b; Ctsk fl/fl osteoclasts (Figure 2F). However, qRT-PCR showed that the efficiency of CD11b-Cre-mediated ablation of Ctsk expression was only $60 \%$ and $61 \%$ in femur and osteoclasts, respectively (Figure 2E). As expected, CD11b-Cre deletion also did not affect expression of Ctsk in osteoblasts (data not shown).

Targeted deletion of Ctsk in hematopoietic cells and osteoclasts increases cancellous bone mass and expression of osteoblast-specific marker genes. We analyzed the skeletal phenotype of 9-week-old Ctskflfl and Mx1;Ctskfl/fl mice. No difference in body weight and bone length was observed between Ctsk $k^{f l f l}$ and $M x 1$; Ctskl/fl mice in the C57BL/6 background, indicating that targeted Ctsk deletion did not affect skeletal development (data not shown). X-ray and micro-CT $(\mu \mathrm{CT})$ analysis of $\mathrm{Cre}^{-}$ and $\mathrm{Cre}^{+}$mice revealed that targeted deletion of Ctsk in hematopoietic cells increased bone mass in femurs (Figure $3 \mathrm{~A}$ ). $\mu \mathrm{CT}$ showed an increase in cancellous bone volume, trabecular number, and connectivity density, with a concomitant decrease in trabecular separation
(Figure 3B). Histomorphometric analysis of distal femurs confirmed a significant increase in cancellous bone volume in both males and females (Figure 4 and Supplemental Table 1; supplemental material available online with this article; doi:10.1172/JCI64840DS1). Analysis of trabecular architecture in $M x 1$; $C t s k^{f l f l}$ mice indicated an increase in trabecular number in males and females, and thickness in females, with a concomitant decrease in trabecular separation in both sexes. While the mineralizing surface was not affected, the mineral apposition rate (MAR) was higher in $M x 1$; Ctsk $f^{l / f l}$ mice, leading to an increase in the BFR. Moreover, osteoblast and osteoclast numbers per tissue area were both markedly increased. As shown in Figure 5A, qRT-PCR indicated that ablation of Ctsk in hematopoietic cells increased the expression of several osteoblast marker genes in femurs, including Runx2, Osterix, ALP, and osteopontin. These data suggest that the high bone mass observed in $M x 1 ; C_{t s k} f^{f / f l}$ mice is likely the consequence of increased bone formation and decreased bone resorption in vivo.

Like $M x 1 ; C t s k^{f l / f l}$ mice, CD11b; $C t s k^{f l / f l}$ mice exhibited a significant $(P<0.05)$ increase in bone mass in the femur at 9 weeks of age, as determined by an increase in cancellous bone volume (Figure 3A) and trabecular thickness using $\mu \mathrm{CT}$ (data not shown). However, $C D 11 b ; C t s k^{f / f l}$ mice had a milder skeletal phenotype than $M x 1 ; C t s k^{f / f l}$ mice. Histomorphometric analysis indicated that CD11b-Creinduced cathepsin $\mathrm{K}$ deficiency increased osteoclast numbers, but also resulted in increases in several indices of bone formation (MAR, BFR, and osteoblast numbers), demonstrating that the increased BFR is osteoclast mediated (Figure 4 and Supplemental Table 2). Femoral cancellous bone volume was increased, but to a lesser extent (29\%) compared with that in Mx1; Ctskflfl mice (78\%). Transcriptional profiling of osteoblast target genes in distal femur metaphysis also showed increased expression of osteoblast marker genes, in particular, Osterix and osteocalcin (Figure 5B).

Deletion of Ctsk in the osteoclast lineage also affects cortical cross-sectional volume and cortical bone volume. In contrast to Mx1;Ctskflffl mice, which showed only a similar trend (Supplemental Table 3 
A
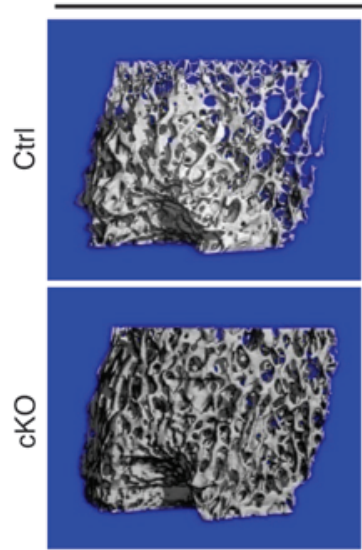

B

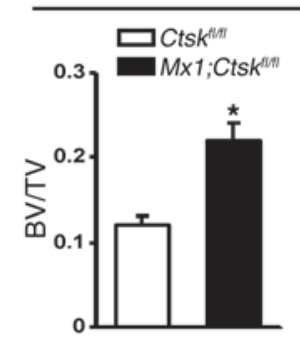

$\mathrm{Mx} 1$
CD11b

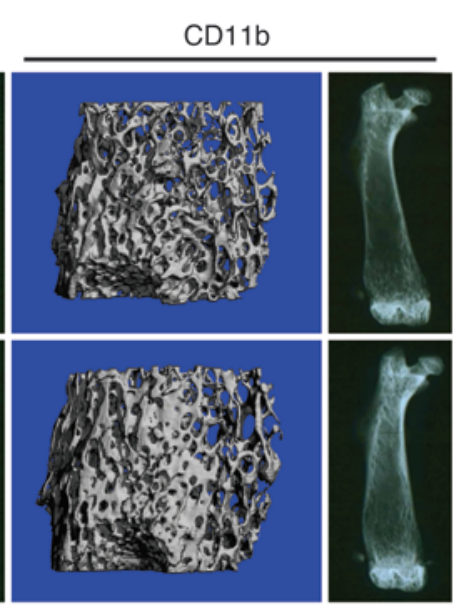

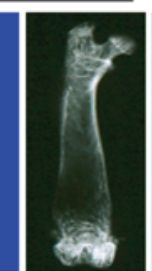

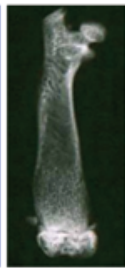

\section{Figure 3}

Deleting Ctsk in hematopoietic cells and osteoclasts increases cancellous bone mass. (A) $\mu \mathrm{CT}$ and $\mathrm{x}$-ray images of femurs from controls (Ctrl) and conditional knockouts (cKO) at 9 weeks of age. (B) $\mu C T$ analysis of distal femur. Results are mean \pm SEM. ${ }^{*} P<0.05$ versus controls.
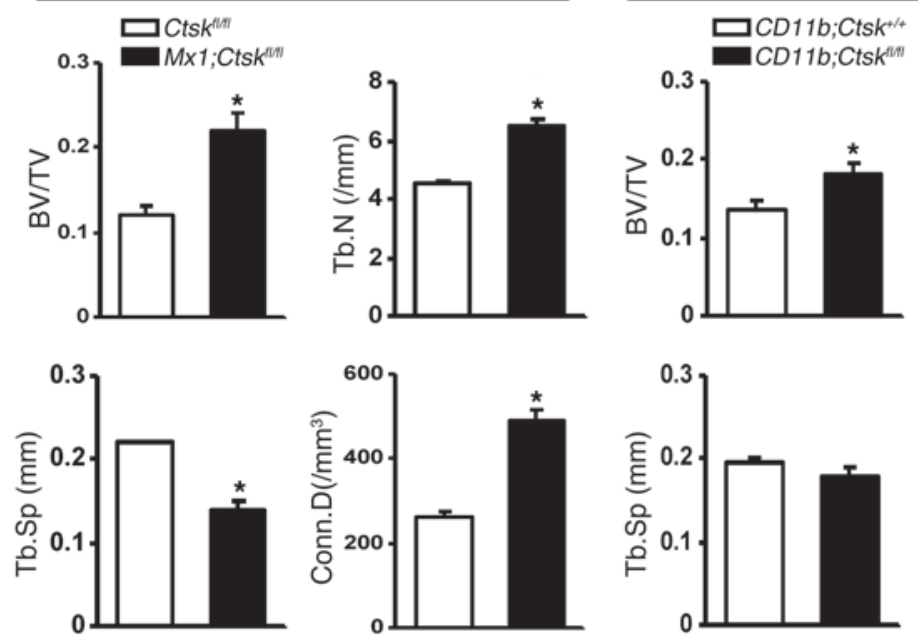

CD11b
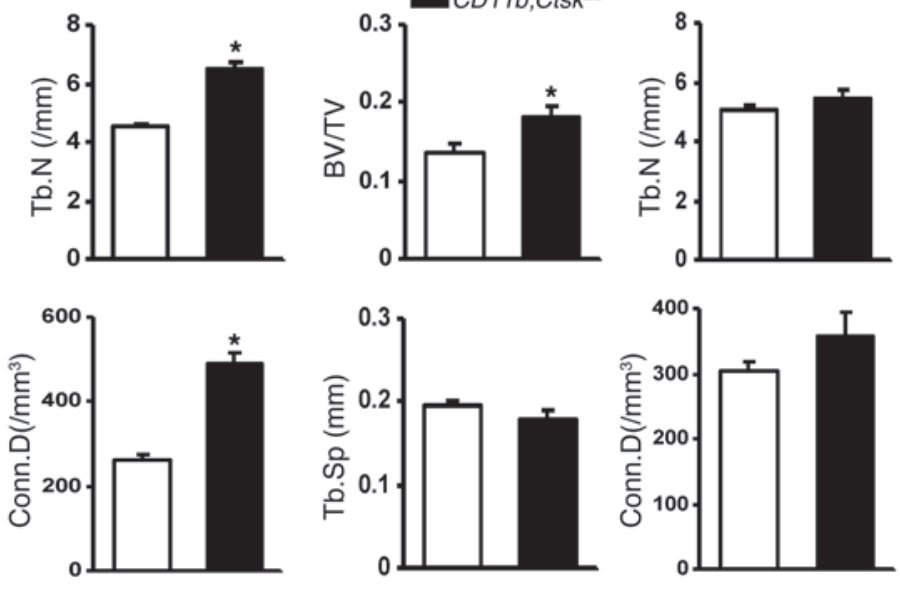

and Supplemental Figure 1), deletion of Ctsk in osteoclasts using the CD11b promoter led to an increase in femoral cross-sectional bone volume and cortical bone volume (Supplemental Table 4 and Supplemental Figure 1), implying an increase in periosteal bone formation after deletion of Ctsk in osteoclasts.

Lack of cathepsin $K$ in hematopoietic cells and osteoclasts impairs bone resorption. To determine whether the increased number of osteoclasts in Mx1;Ctskfl/fl and CD11b;Ctsk $k^{f l f l}$ mice was cell autonomous or secondary to changes occurring in other cells, we analyzed osteoclast differentiation in vitro. Mx1-Cre-induced deletion of Ctsk in hematopoietic cells and osteoclasts increased osteoclast differentiation from BM macrophages (BMMs) (Figure 6, A and B). We plated osteoclasts on dentin slices and compared their resorbing ability to examine whether defective osteoclast function contributed to the increased bone volume in $M x 1 ; C t s k f / f l$ and CD11b; Ctsk $k^{f / f l}$ mice. We found that resorption pit depth was reduced, such that the pits formed by Mx1; Ctskflfl osteoclasts were shallow (Figure $6 \mathrm{C})$. Immunofluorescence labeling of phalloidin confirmed that $M x 1$; $C t k^{f l / f l}$ osteoclasts formed a flat, actin-rich sealing zone (Supplemental Figure 2). It has been shown that deletion of Ctsk and treatment with cathepsin $\mathrm{K}$ inhibitors interrupts intracellular vesicle trafficking, leading to an accumulation of type I collagen-containing vesicles and reduced bone resorption effi- ciency $(17,26)$. Immunofluorescence staining of synaptotagmin 7 , a marker of "secretory" lysosomes (27), showed an accumulation of labeled vesicles in Ctsk-deleted osteoclasts (Figure 7A). Similarly, vesicles labeled for the lysosome-associated membrane protein 2 (LAMP2), a late endosomal-lysosomal marker, also accumulated in Ctsk-deleted osteoclasts (Figure 7B). This is consistent with the reports indicating that deletion or inhibition of cathepsin $\mathrm{K}$ alters normal intracellular vesicle trafficking $(17,26)$, possibly further altering osteoclast function. Thus, the absence of cathepsin $\mathrm{K}$ in osteoclasts increased their numbers, but decreased their bone-resorbing activity, leading to an increase in bone mass.

Targeted deletion of Ctsk in the osteoblast lineage has no effect on bone formation. Although the level of expression of Ctsk is much lower in osteoblasts than in cells of the osteoclast lineage, this enzyme could still play an intrinsic role in osteoblasts, possibly affecting bone formation. To examine whether the increased BFR in global or targeted Ctsk-deficient mice could be due to the absence of cathepsin $\mathrm{K}$ in osteoblasts, we generated mice with Ctsk conditionally deleted in cells of the osteoblast lineage by crossing Ctsk $\mathrm{fl}^{\mathrm{l}+\mathrm{+}}$ mice with Osx-Cre mice, thereby targeting expression of Cre to the osteoblast lineage at early stages of differentiation. Since Osx-Cre mice are mildly osteopenic (28), we compared Osx; $\mathrm{Ctsk}^{+/+}$(controls) with Osx;Ctsklffl mice (conditional knockouts). We found no 

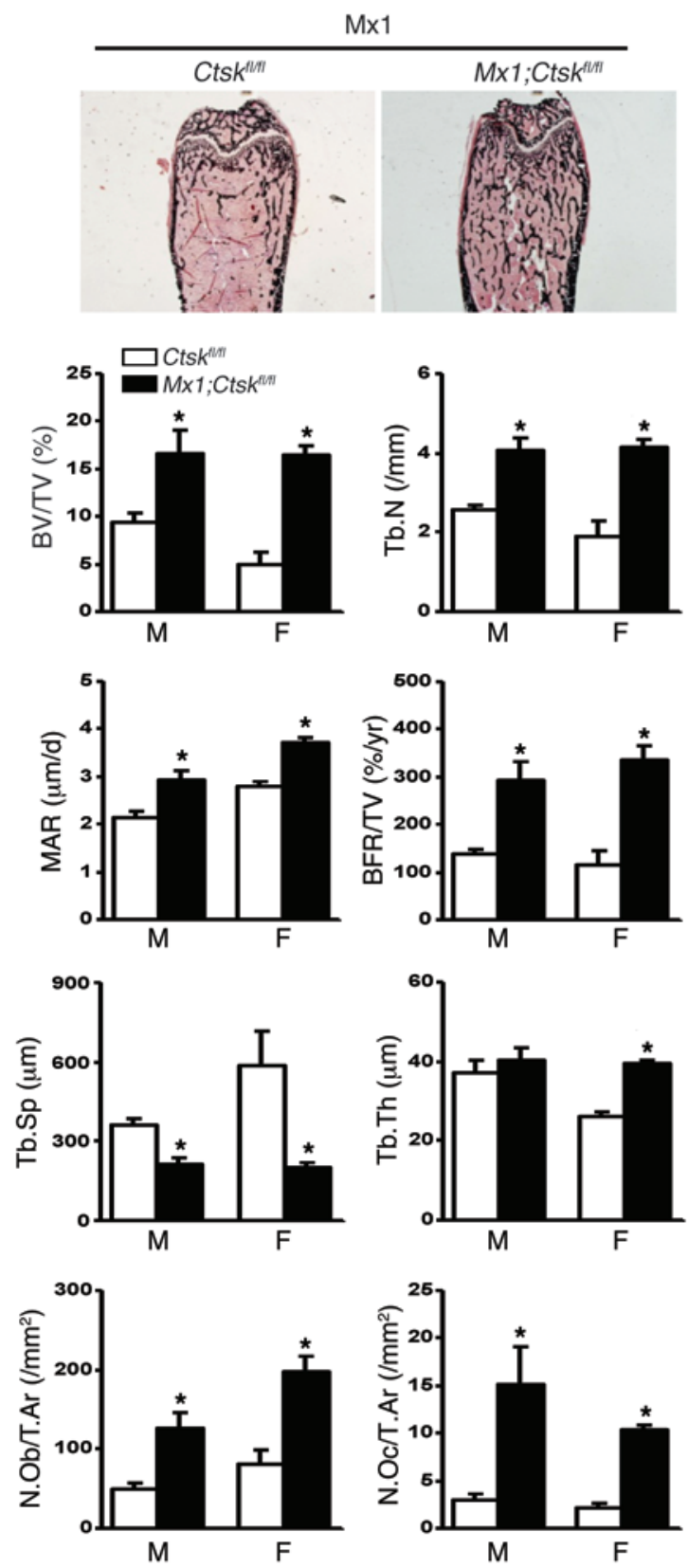
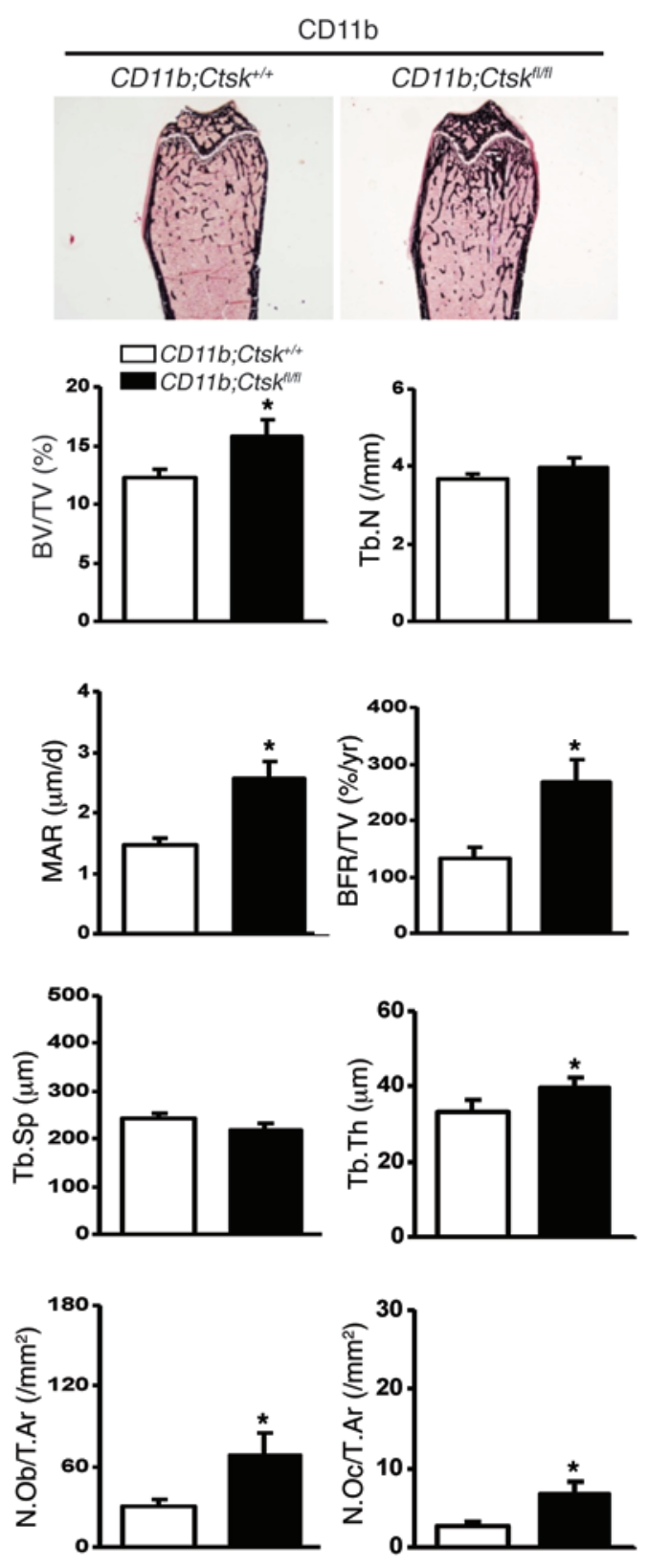

Figure 4

Deleting Ctsk in hematopoietic cells and osteoclasts increases cancellous bone mass, bone formation, and numbers of osteoblasts and osteoclasts. Histomorphometric analysis of cancellous bone in femurs from 9 -week-old mice. Results are mean \pm SEM. ${ }^{\star} P<0.05$ versus controls. Original magnification, $\times 2$.

changes in bone density at 5, 9, or 13 weeks of age by x-ray; data from 13 -week-old mice are presented. $\mu \mathrm{CT}$ analysis showed no difference in cancellous and cortical bone volume at 13 weeks of age (Figure 8, A and B, Supplemental Table 5, and Supplemental Figure 1). Furthermore, histomorphometric analysis demonstrated no alterations of the skeletal phenotype in osteoblast-targeted Ctsk-deficient mice (Figure 8C and Supplemental Table 6). In contrast to $\mathrm{M} x 1$-Cre- or CD11b-Cre-driven deletion of Ctsk, deletion of Ctsk in the osteoblast lineage had no effect on bone volume, $\mathrm{BFR}$, osteoblast numbers, or osteoclast numbers. In vitro assays of primary osteoblasts derived from the long bones showed that ALP and mineralized bone nodules were similar in cells derived from Osx; Ctsk $f^{f l / l}$ mice and their control littermates (Figure 8D). These data demonstrate that the bone volume and BFR changes observed in $M x 1 ; C t s k{ }^{f l f l}$ or CD11b; Ctsk ${ }^{f l f l}$ mice were not due to the deletion of Ctsk in osteoblasts. Therefore, the observed increases in BFR in Mx1-Cre and CD11b-Cre-deleted cathepsin K mice are secondary to the deletion of Ctsk in cells of the osteoclast lineage.

Lack of Ctsk in hematopoietic cells increases osteoblast precursors. Since Cre-induced deletion of Ctsk using the CD11b promoter was less 

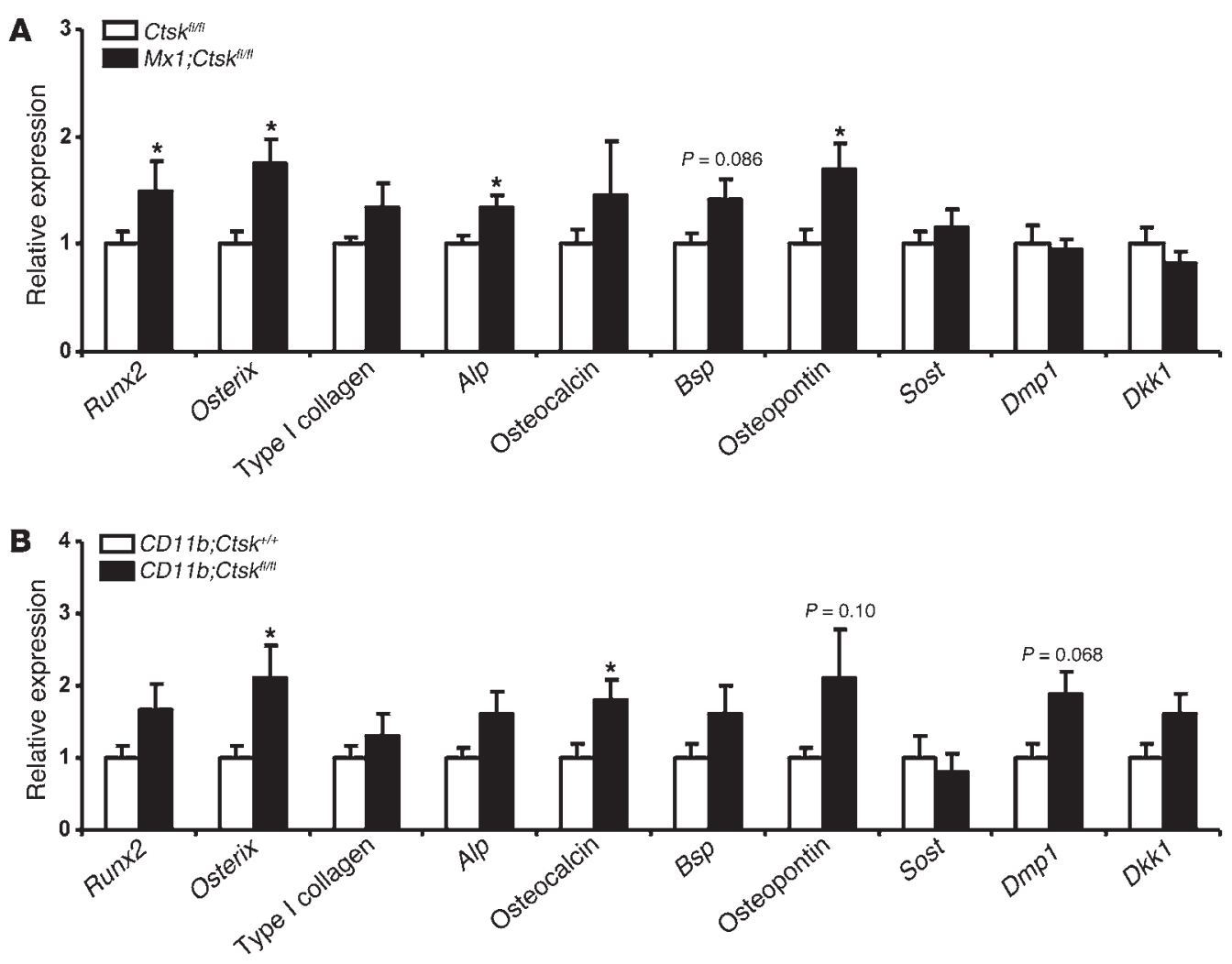

\section{Figure 5}

Osteoblast marker genes are upregulated in distal femur metaphysis of $M \times 1 ; C t s k^{f l|f|}$ and $C D 11 b ;$ Ctsk $^{f l / f l}$ mice. (A) qRT-PCR analysis of mRNA expression in distal femur metaphysis from 9-weekold Ctsk $k^{f l / f l}$ and $M \times 1 ;$ Ctsk $^{f \mid l f l}$ mice. (B) qRT-PCR analysis of mRNA expression in distal femur metaphysis from 9-week-old CD11b;Ctsk ${ }^{+/+}$ and $C D 11 b ; C t s k^{f / f l}$ mice. Data are expressed as percentage of mRNA level in controls. Results are mean \pm SEM. ${ }^{*} P<0.05$ versus controls. efficient than the deletion obtained with the Mx1-Cre promoter, generating a qualitatively similar but milder skeletal phenotype, we used $M x 1$; $C t k^{f l / f l}$ mice to further characterize the mechanism by which Ctsk deficiency in osteoclasts led to an increase in BFR. To determine whether the increased bone formation observed in $M x 1$; Ctskflfl mice was due to an increased commitment of osteoblast progenitors, we examined their proliferation and differentiation in long bone-derived primary osteoblasts in vitro. Osteoblasts derived from long bones of $M x 1$; $C t s k^{f l / f l}$ mice had more ALP activity and formed more mineralized bone nodules than did control cells (Figure 9A). In addition, ablation of Ctsk increased CFU-ALP and CFU osteoblasts (CFU-OBs), but not CFU fibroblasts (CFU-Fs) (Supplemental Figure 3). Consistent with the CFU-F assay, BrdU labeling showed no change in osteoblast proliferation between $M x 1$; Ctskfl/fl osteoblasts and controls (Supplemental Figure 3). These observations suggest that the lack of cathep$\sin \mathrm{K}$ in hematopoietic cells increased the number of committed osteoblast progenitors in bone, contributing to increased bone formation in vivo and in vitro. Consistent with these in vitro findings, FACS analysis confirmed a $17 \%$ increase in ALP-positive cells derived from $M x 1$; $C t s k^{f l f l}$ long bone osteoblasts compared with controls (Figure 9B). To analyze the differentiation of $M x 1$; Cts $k^{f / f l}$ osteoblasts, we isolated total RNA from osteoblasts derived from long bone of $M x 1 ; C t s k^{f l / f l}$ mice and their control littermates, and the expression of osteoblast marker genes was analyzed by qRTPCR (Figure 9C). Expression of several markers of osteoblast differentiation ( $A L P$, type I collagen, Runx2, and Dmp1) was significantly upregulated in $M x 1$; Ctskflfl cells. Osteopontin mRNA expression $(P=0.064)$ was slightly increased in Mx1; Ctskfl/fl osteoblasts, but did not reach significance. In addition, RANKL mRNA was markedly increased, whereas $O P G \mathrm{mRNA}$ levels decreased, leading to a significant increase in the ratio of RANKL and $O P G$ mRNA levels. Consistent with the increase in $R A N K L / O P G$ ratio, we observed greater numbers of TRAP-positive osteoclasts formed in coculture of Mx1;Ctskflfl osteoblasts with Ctsk ${ }^{f l / f l} \mathrm{BMMs}$ (Figure 9D). Taken together, these in vitro results show that ablation of Ctsk in the osteoclast lineage: (a) increases the number and differentiation of cells of the osteoblast lineage, and (b) increases the RANKL/OPG ratio in these cells, leading to an increase in the number of osteoclasts, as shown in vivo and in vitro.

Deletion of Ctsk increases Sphk1 expression in osteoclasts, and S1P in osteoclast-conditioned medium increases ALP in osteoblasts. Since Osx; $C t s k^{f l / f l}$ mice do not have a skeletal phenotype, we hypothesized that reducing Ctsk expression in osteoclasts resulted in more anabolic coupling from osteoclasts to osteoblasts, and thus increased remodeling-based bone formation. To test this hypothesis, we first determined whether conditioned medium from $M \times 1$; Ctsk $f^{l / f l}$ and CD11b; $C t s k^{f l / f l}$ osteoclasts increased ALP in osteoblasts. CD1 calvarial osteoblasts were cultured in conditioned medium from either knockout or control osteoclasts. As shown in Figure 10, calvarial osteoblasts treated with conditioned medium derived from $M x 1 ; C t s k^{l / f l}$ and CD11b; Ctsk fl/fl osteoclast cultures showed increased ALP staining, indicating that the conditioned medium contained more osteoanabolic factors.

To identify coupling factors that might be increased in the Ctsk-null osteoclasts, we measured in vitro gene expression of the known osteoclast-derived coupling factors Efnb2, Wnt10b, Bmp6, and SPHKs (which generates S1P) $(20,29-31)$ in osteoclasts generated from the $M \times 1 ; C t s k^{f l f l}$ and $C D 11 b ; C t s k^{f l / f l}$ mice. Ablation of Ctsk in hematopoietic cells and osteoclasts increased Spbk1 mRNA expression, but not Sphk2, Efnb2, Wnt10b, or Bmp6 expression (Figure 11A). Immunostaining confirmed the increased SPHK1 
A

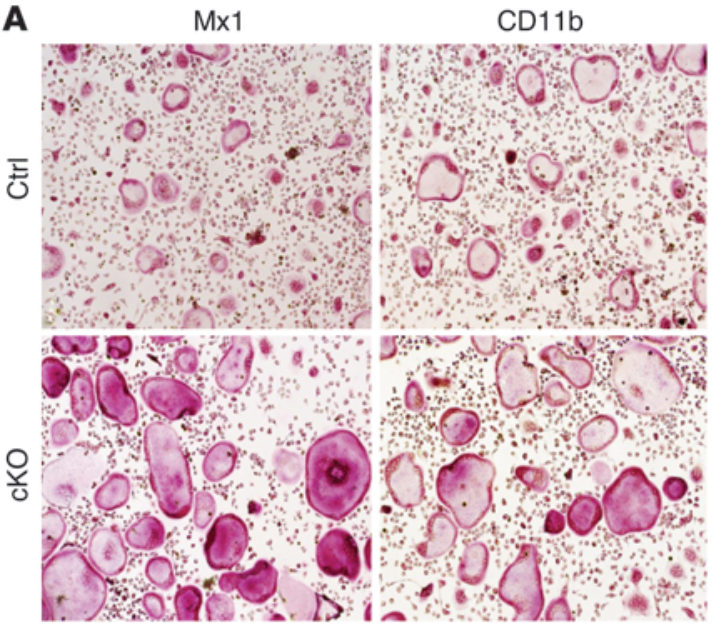

C

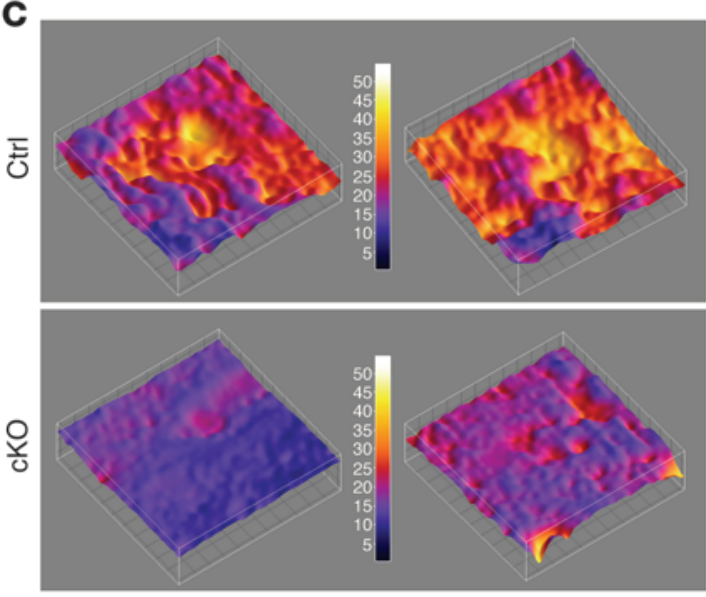

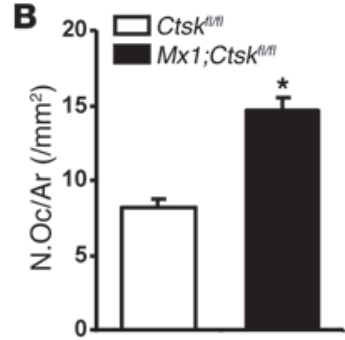

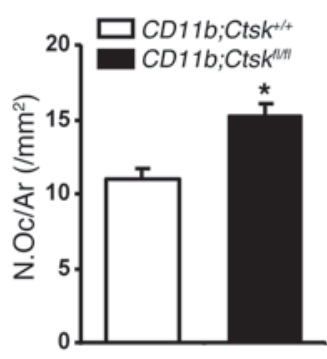

Figure 6

Targeted deletion of Ctsk in hematopoietic cells and osteoclasts increases osteoclast differentiation and decreases bone resorption. (A) Control (Ctrl) and conditional knockout (CKO) osteoclasts were generated on glass coverslips in the presence of M-CSF and RANKL. Original magnification, $\times 10$. (B) TRAP-positive osteoclast numbers per area $\left(/ \mathrm{mm}^{2}\right)$ were quantified by OsteoMeasure software (OsteoMetrics). Results are mean \pm SEM. ${ }^{\star} P<0.05$ versus controls. (C) Osteoclasts generated by coculture with osteoblasts on collagen gel in the presence of vitamin $\mathrm{D}_{3}$ and $\mathrm{PGE}_{2}$ were replated onto dentin slices and cultured for 48 hours. Images of resorption pits on dentin slices resorbed by control and conditional knockout osteoclasts were analyzed by Fuji software.

ditioned medium (Figure 12, B and C). These data confirm that S1P is a major contributor to the Ctsk-deficient osteoclast-mediated enhancement of bone formation in vitro.

\section{Discussion}

Our results establish several important findings regarding bone remodeling. First, our genetic studies show that bone formation is regulated, in part, by cells of the osteoclast lineage, experimentally providing evidence for a coupling mechanism in an in vivo setting. Second, in vitro studies have established S1P as a key osteoclast-derived coupling messenger, a "clastokine" synthesized and secreted by osteoclasts that affects osteoblast differentiation and acts locally to promote the osteoblasts'

in Mx1;Ctsk $k^{f l f l}$ and CD11b; Ctsk $k^{f l f l}$ osteoclasts plated on dentin slices (Figure 11B). We also determined SPHK1 protein levels by Western blotting (Figure 11C). Consistent with the mRNA analysis, Ctsk-deficient osteoclasts had higher SPHK1 protein levels. Sphk1 catalyzes the phosphorylation of sphingosine to S1P, which has been reported to target osteoblasts and increase bone formation (20). We therefore examined the levels of S1P in Mx1;Ctsk $\mathrm{fl}^{\mathrm{f} / \mathrm{l}}$ osteoclast-conditioned medium using mass spectrometry. The conditioned medium from $M x 1 ; C t s k^{l / f l}$ osteoclasts contained significantly more S1P than Ctsk ${ }^{f / f l}$ osteoclast-conditioned medium (Figure 12A). Interestingly, immunofluorescence staining of Sphk1 was not increased in $\mathrm{Src}^{-1-}$ osteoclasts (Supplemental Figure 4), indicating that it was not the result of osteoclast functional impairment, but rather was a specific effect of Ctsk deletion.

Antagonizing S1P reduces Mx1;Ctskl/fl osteoclast-conditioned medium-induced ALP activity and mineralization. Mouse osteoblasts express three $\mathrm{S} 1 \mathrm{P}$ receptors: $\mathrm{S}_{1} \mathrm{P}_{1}, \mathrm{~S}_{1} \mathrm{P}_{2}$, and $\mathrm{S}_{1} \mathrm{P}_{3}(20)$. To further verify whether biologically active $\mathrm{S} 1 \mathrm{P}$ is responsible for the coupling-mediated increase in bone formation, VPC23019, an $\mathrm{S}_{1} \mathrm{P}_{1,3}$ antagonist, was added to the cultures of CD1 calvarial osteoblasts in the presence of conditioned medium from either Mx1;Ctsk $\mathrm{fl}^{\mathrm{f} / \mathrm{fl}}$ or Ctsk $k^{f / f l}$ osteoclast cultures. VPC23019 at $2 \mu \mathrm{M}$ did not have any measurable effect on ALP activity (data not shown), but $10 \mu \mathrm{M}$ VPC23019 markedly inhibited the increase in ALP activity and mineralization that are induced by $M x 1 ; C t s k^{f / f l}$ osteoclast-con- bone-forming activity. Third, our studies indicate that the presence and/or activity of cathepsin $\mathrm{K}$ within cells of the osteoclast lineage represses the expression of Sphk1, and thereby the synthesis of S1P, decreasing the ability of osteoclasts to stimulate bone formation. As a result, deleting or decreasing cathepsin $\mathrm{K}$ expression in osteoclasts favors S1P synthesis and bone formation. Accordingly, blocking $\mathrm{S}_{1} \mathrm{P}_{1,3}$ in osteoblast cultures decreases osteoclast-conditioned medium-induced ALP activity and mineralization. Fourth, these studies suggest that cells of the osteoblast lineage attempt to compensate for the functional defect of individual osteoclasts by increasing their numbers through activation of the RANKL pathway, possibly also in response to increased S1P levels (20). Taken together, these results support the hypothesis that therapeutic manipulation of osteoclast activity via inhibition of cathepsin $\mathrm{K}$ can decrease bone resorption, while maintaining or increasing bone formation at remodeling sites. Finally, our finding of an increase in total cross-sectional volume and cortical bone volume, without any change in marrow volume after deletion of Ctsk in cells of the osteoclast lineage, suggest that it may also indirectly affect periosteal modeling-based bone formation, an observation also made after pharmacological inhibition of cathepsin $\mathrm{K}$ in nonhuman primates $(32,33)$.

The adult skeleton undergoes constant remodeling throughout life. The remodeling process begins with resorption by multinucleated osteoclasts, followed by osteoblast-mediated bone formation. 
A
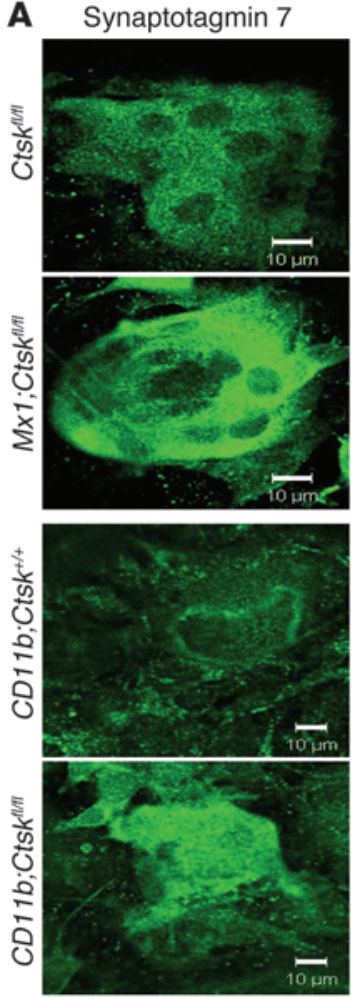

B
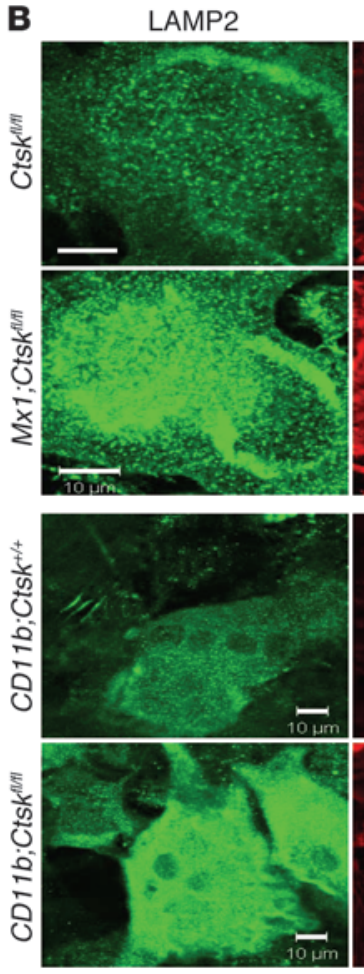

Actin
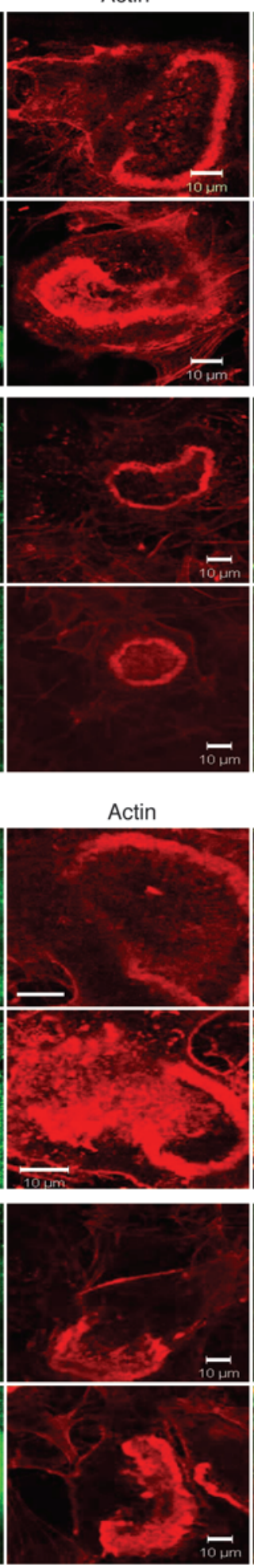

Merge
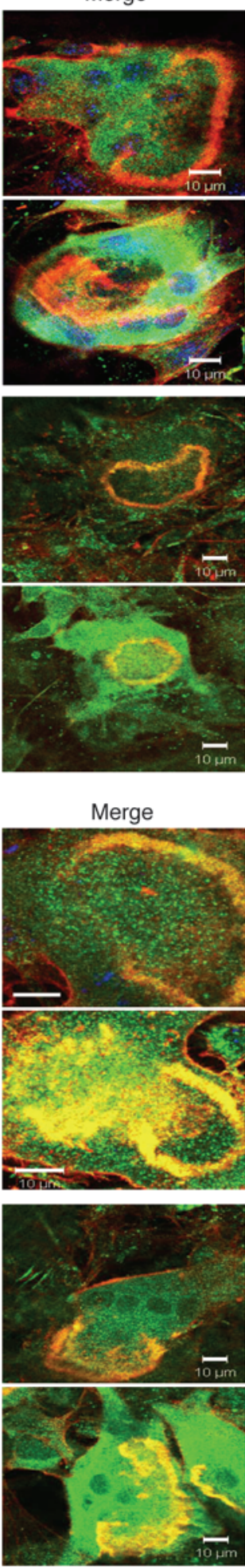

\section{Figure 7}

Deletion of Ctsk in hematopoietic cells and osteoclasts increases accumulation of intracellular lysosomes. Osteoclasts generated by coculture with osteoblasts on collagen gel in the presence of vitamin $D_{3}$ and $P G E_{2}$ were replated onto dentin slices and cultured for 48 hours. (A) Fluorescence micrographs of osteoclasts showing synaptotagmin 7 (green) and actin (red). (B) The localization of LAMP2 (green) and actin (red) was analyzed in control and conditional knockout osteoclasts. Scale bar: $10 \mu \mathrm{m}$.

to inhibit bone resorption through antiresorptive drugs. As a consequence of the coupling process, inhibition of bone resorption generally leads to a parallel decrease in bone formation. This, in turn, leads to low bone turnover and may contribute to unwanted consequences such as ONJ or atypical femoral fractures. In contrast, PTH, which is the only approved anabolic drug for the treatment of osteoporosis, increases bone formation, but secondarily leads to increased bone resorption. The secondary increase in resorption induced by PTH is due to increased RANKL production by osteoblasts, the direct targets of PTH (34). These observations, made both in animal studies and human clinical trials, have led to the conclusion that bone mass could be gained only during limited periods of time (the so-called "anabolic windows") until bone formation decreases after antiresorptive treatment, or until bone resorption increases after PTH treatment.

Global loss of cathepsin K leads to an osteopetrotic phenotype due to defective bone resorption that is characterized in mice by an increase in the number of poorly functional osteoclasts and increased bone formation $(18,19)$. The present study was aimed at understanding the molecular mechanisms by which such a potentially beneficial dissociation of bone resorption and bone formation was induced by the deletion of Ctsk. We initially demonstrated that the phenotype of the germline deletion of Ctsk was reproduced by deleting Ctsk in osteoclasts using Mx1-Cre. However, Mx1-Cre targets multiple hematopoietic cell types, including megakaryocytes, lymphocytes, mast cells, and cells of the monocyte-macrophage lineage, and we could not rule out possible cathepsin $\mathrm{K}$-dependent contributions to regulation of bone homeostasis by other types of cathepsin K-expressing hematopoietic cells in this mouse model. To confirm that the altered bone homeostasis was a consequence of deleting Ctsk in osteoclasts, we used a more specific conditional knockout of CD11b-Cre for targeting Cre recombinase exclusively to the monocyte-macrophage lineage. The skeletal phenotype of CD11b; Ctsk fl/fl mice was identical, albeit milder, and was probably due to the lower efficiency of Ctsk deletion in CD11b; Ctsk $k^{f / f l}$ osteoclasts than that in $M x 1 ; C t s k^{l / f l}$ mice. The smaller increase

To maintain skeletal homeostasis, bone resorption is coupled to bone formation, and imbalances in this process lead to a variety of human diseases including osteoporosis and osteopetrosis. Osteoporosis is the result of an excess of bone resorption over bone formation during the remodeling process. The most common approach for the treatment of this debilitating disease has been in bone volume in CD11b; Ctsk fl/fl mice than that seen in Mx1; Ctsk $k^{f / f l}$ mice (29\% vs. $78 \%$ ), notwithstanding the similar increases in MAR and BFR, is likely due to the greater residual bone-resorbing activ-

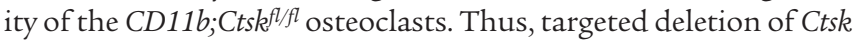
in cells of the osteoclast lineage resulted in a skeletal phenotype comparable to the global Ctsk knockouts. 

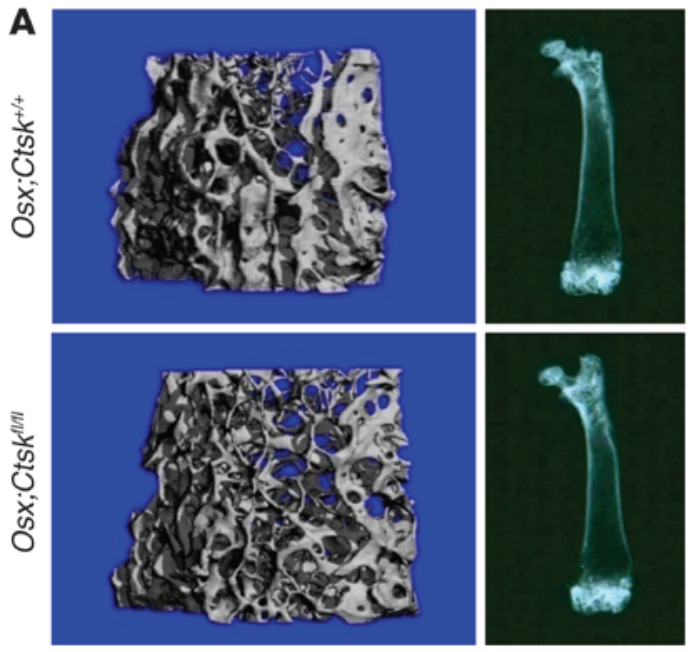

C
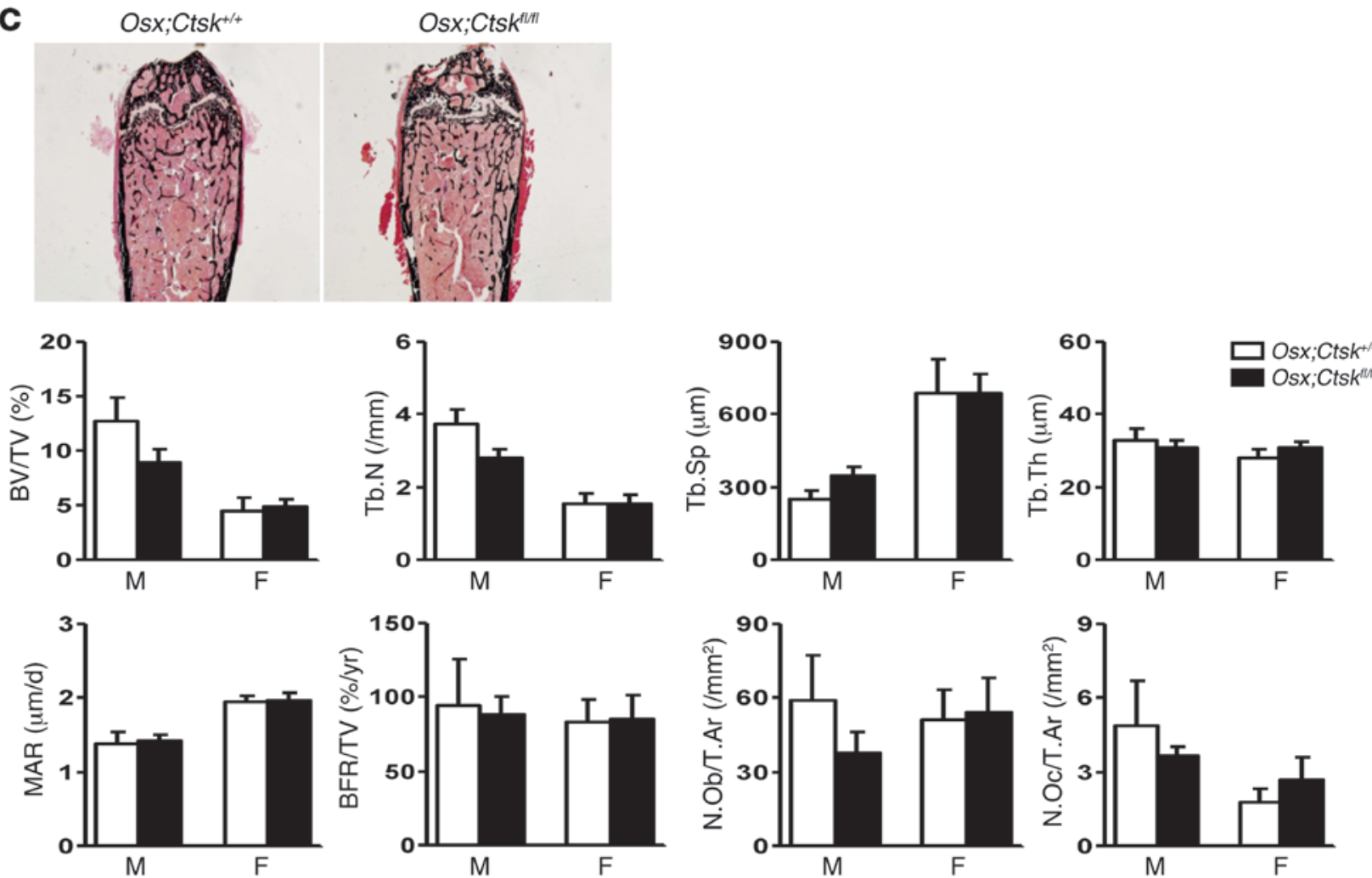

D

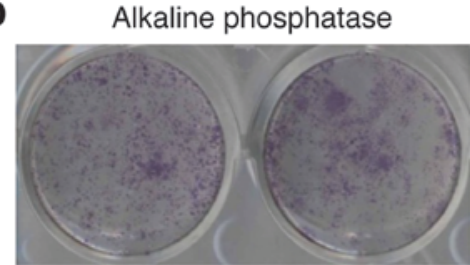

Osx $; \mathrm{Ctsk}^{+/+}$

Osx:Ctsk'
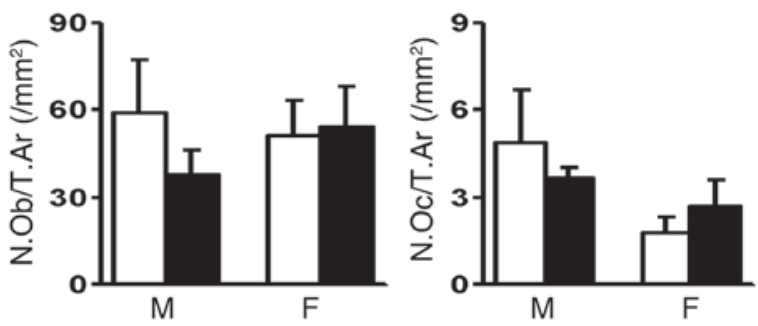

Alizarin red

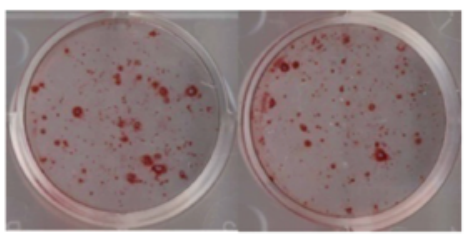

Osx $; \mathrm{Ctsk}^{+/+}$

Osx:Ctsk

Figure 8

Deletion of Ctsk in cells of osteoblast lineage has no effect on bone formation and bone resorption. (A) $\mu \mathrm{CT}$ and $\mathrm{x}$-ray images of femurs from Osx; $\mathrm{Ctsk}^{+/+}$and Osx;Ctsk ${ }^{\text {fllfI }}$ mice at 13 weeks of age. (B) $\mu \mathrm{CT}$ analysis of distal femur metaphysis. (C) Histomorphometric analysis of cancellous bone in femurs from 13-week-old Osx; Ctsk $k^{f l f t}$ mice and controls. Original magnification, $\times 2$. (D) ALP and mineralized nodule in osteoblasts derived from long bones of Osx; $\mathrm{Ctsk}^{+/+}$and Osx;Ctsk ${ }^{\mathrm{tl} / \mathrm{ll}}$ mice. Results are mean $\pm \mathrm{SEM}$. 
A

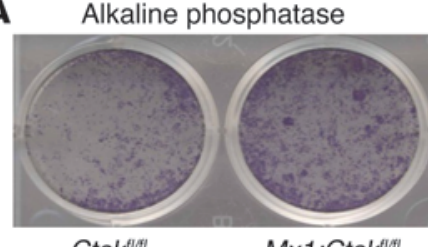

Ctsk $k^{\text {IIII }}$

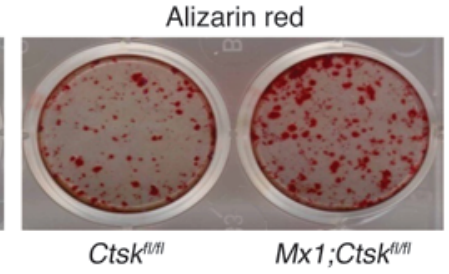

B

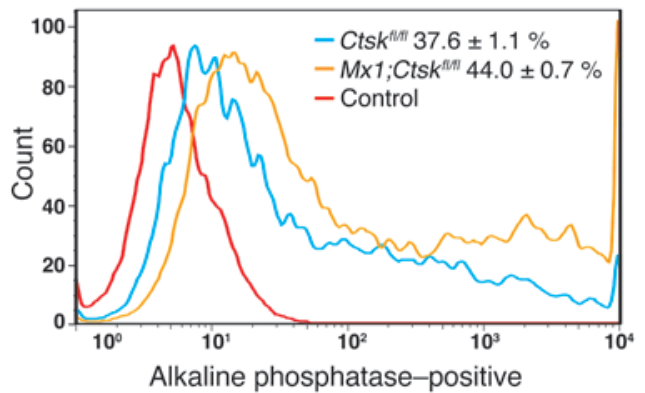

C

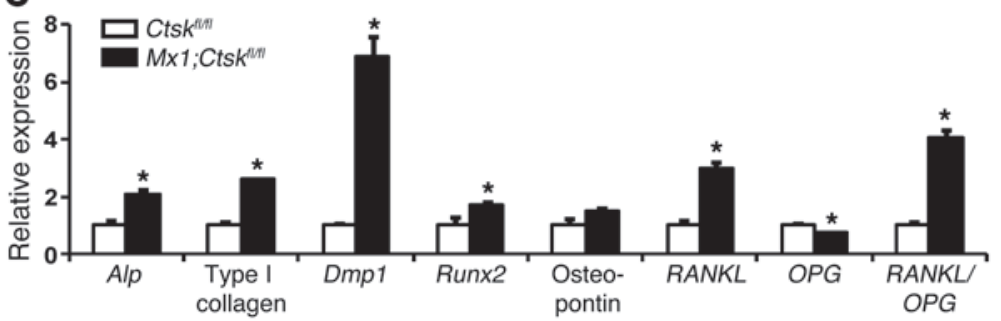

D
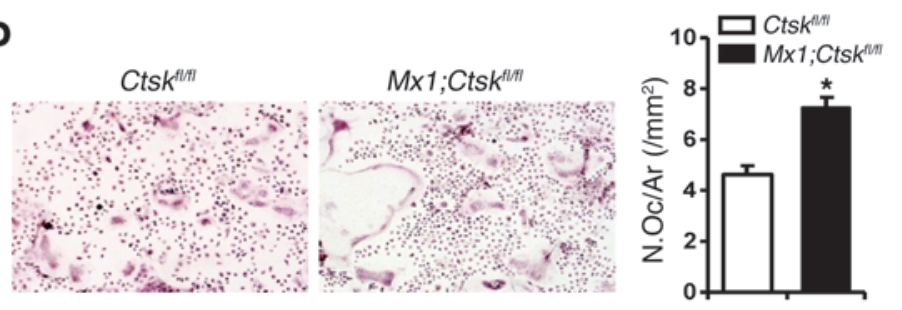

\section{Figure 9}

Genetic deletion of Ctsk in hematopoietic cells increases committed osteoprogenitors. (A) ALP and mineralized nodule in osteoblasts derived from long bones of $M \times 1 ; C t s k^{f / f t}$ mice and their control littermates. (B) FACS analysis of the percentage of ALP-positive cells from long bone-derived osteoblasts of $M \times 1 ; C t s k^{f \mid l t l}$ mice (yellow; $n=4$ ) and Ctsk $k^{\text {flftl }}$ (blue; $n=4$ ) mice. Cells were stained with either PE-labeled anti-ALP or PE-labeled isotype control (red). (C) qRT-PCR analysis of mRNA expression in culture osteoblasts derived from long bones of Ctsk ${ }^{f l f f l}$ mice $(n=3)$ and Mx1;Ctsk $k^{f l f l}$ mice $(n=3)$. Data are expressed as percentage of mRNA level of controls. (D) TRAP-positive osteoclast number per area $\left(/ \mathrm{mm}^{2}\right)$ from coculture of either Ctsk ${ }^{f l / f l}$ or $M x 1 ; C t s k^{f / f l}$ osteoblasts with Ctsk ${ }^{\text {fl/fl }} \mathrm{BMMs}$. Results are mean \pm SEM. ${ }^{\star} P<0.05$ versus controls.

bers of CFU-ALP and CFU-OBs without any changes in total CFU-Fs.

Within bone remodeling sites, the transition from bone resorption to bone formation occurs as a result of coupling activity mediated by several factors. In recent years, several coupling factors have been identified that can be classified in 2 categories: (a) matrix-derived growth factors (MDGFs) that are released from bone matrix during the bone resorption process, and (b) osteoclast-derived cytokines (clastokines) that are soluble factors secreted by osteoclasts into the local microenvironment, or membrane-bound proteins that mediate bidirectional signaling between osteoblasts and osteoclasts $(11,20,29-31,38)$. Ctsk deletion appears to favor coupling through alteration in both categories of coupling factors (Figure 13, A and B). Fuller and colleagues have shown in vitro that the decreased degradation of collagen and other bone matrix proteins when cathepsin $\mathrm{K}$ is inhibited increases the levels of intact IGF-1 released during bone resorption by osteoclasts, potentially enhancing local bone forma-

It has been reported that cathepsin $\mathrm{K}$ is expressed in mesenchymal stem cells, osteoblasts, osteocytes, and bone marrow stromal cells (BMSCs) in humans, albeit at much lower levels than in osteoclasts (35-37). The role of cathepsin $\mathrm{K}$ in human osteoblasts is unclear. Our data demonstrate that it does not appear to affect bone remodeling and homeostasis, since mice with osteoblast-targeted disruption of cathepsin K using Osx-Cre did not exhibit any skeletal phenotype, indicating that decreasing the already low levels of Ctsk expression in cells of the osteoblast lineage does not contribute to the increased bone formation observed in the globally Ctsk-deleted mice. Thus, it appears that inhibition of cathepsin $\mathrm{K}$ leads to increased cancellous bone formation through indirect osteoclast-mediated effects and not through mesenchymal or osteoblast lineage cell-autonomous effects in mice. The fact that osteoblasts isolated from the osteoclast-targeted conditional knockout mice form more bone nodules in vitro and the number of osteoblasts in bone increases, suggests that in vivo changes occurring in cells of the osteoblast lineage cells after deletion of Ctsk in osteoclasts result from an increase in the number of committed osteoblast precursors. This conclusion is supported by our observation of increased numbers of ALP-positive cells by FACS analysis, as well as increased num- tion (11). Similarly, inhibition of cathepsin K may locally increase the levels of activated TGF- $\beta$, which is released from bone matrix during osteoclastic bone resorption and has recently been shown to induce migration of osteoblast precursors to bone remodeling sites, thus favoring bone formation (38). Although all these studies were performed in vitro and no information is available in vivo, our studies cannot exclude the possibility that MDGFs contribute to the phenotype we observed here in vivo in osteoclast-targeted Ctsk deletions. Notwithstanding this possibility, the finding that conditioned medium derived from $M x 1 ; C t s k l / f l$ or CD11; Ctsk $f l / f l$ osteoclasts cultured in the absence of bone matrix increased ALP in osteoblasts demonstrates that osteoclast-secreted anabolic signals that are independent of resorptive activity are increased after deletion of Ctsk.

We have identified S1P, a known clastokine (20, 29-31), as a factor derived from osteoclasts and as being responsible for the observed phenotype. S1P is produced by sphingosine kinase in osteoclasts and interacts with the $\mathrm{S} 1 \mathrm{P}$ receptors expressed on osteoblasts, enhancing migration and survival of osteoblasts as well as RANKL expression (20). Others have shown by microarray analysis that increased expression of Sphk1 by osteoclasts correlates with enhanced osteoblast differentiation in vitro (29). We 

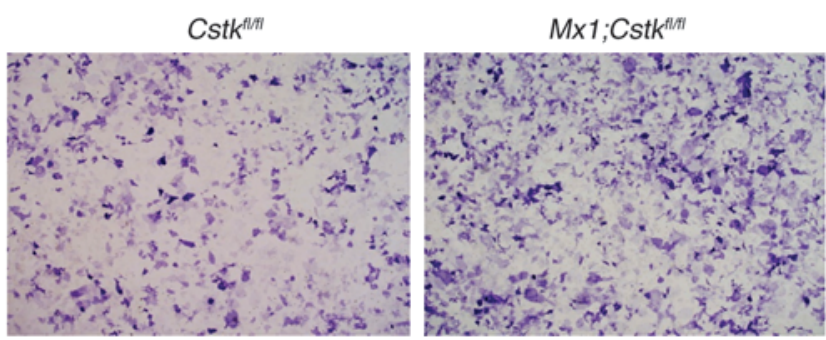

CD11b;Cstk ${ }^{+/+}$
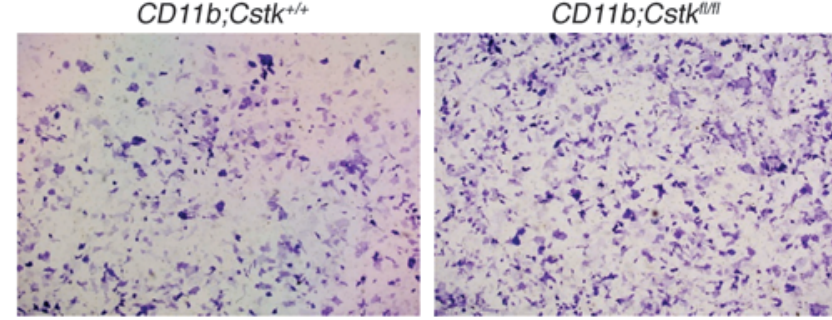

Figure 10

Conditional knockout osteoclast-conditioned medium increases ALP staining. CD1 calvarial osteoblasts were treated with either control or conditional knockout osteoclast-conditioned medium for 7 days and stained with ALP. Original magnification, $\times 2$.

found that Sphk1 expression was upregulated in $M x 1$; Ctsk $f^{f l f l}$ and $C D 11 b$-Ctsk ${ }^{f / f l}$ osteoclasts, and the S1P content of Mx1; Ctsk $f^{f / f l}$ osteoclast-conditioned medium was increased. In addition, an $\mathrm{S}_{1} \mathrm{P}_{1,3}$ antagonist blunted the anabolic effects of osteoclast-conditioned medium in vitro. The upregulated Sphk1 in individual Ctsk-deficient osteoclasts and the consequent increase in S1P production, together with the increased osteoblast differentiation induced by the $M \times 1 ; C t s k^{f / / f l}$ osteoclast-conditioned medium and the increased bone formation in mice in vivo, confirm that osteoclasts can affect BFR via secreted molecules acting on cells of the osteoblast lineage. Thus, S1P appears to be an important coupling factor, and our data indicate that cathepsin $\mathrm{K}$ acts to decrease its production.

Others have shown that extracellular S1P stimulates osteoclastogenesis in coculture of BMMs and osteoblasts by increasing RANKL production, but has no effect on osteoclast differentiation in BMM cultures treated with RANKL (20). Consistent with this mechanism, we observed a significant increase in $R A N K L$ expression in osteoblasts derived from long bones in $M \times 1$; Ctsk $\mathrm{fl}^{\mathrm{f} f l}$ mice. Since cells of the osteoblast lineage, including osteocytes, are the major source of RANKL in mice $(39,40)$, this implies that changes occurring in cathepsin $\mathrm{K}$-deficient osteoclasts led to this osteoblast-mediated response, forming a positive feedback loop to promote bone resorption by increasing osteoclastogenesis. It is therefore likely that osteoclast-derived S1P is responsible not only for the increased BFR observed in vivo and ALP activity observed in vitro, but also for the stimulation of RANKL production by cells of the osteoblast lineage that leads to the increased osteoclastogenesis observed in both osteoclast-targeted and global Ctsk deletions.

Our observation that in vitro osteoclastogenesis is increased in BMMs derived from conditional knockout mice in response to M-CSF and RANKL raises an interesting additional question, and suggests that the increased osteoblast-derived RANKL/OPG ratio may not be the only explanation for the greater number of osteoclasts observed in vivo. As the cells in this assay are exposed to equal levels of M-CSF and RANKL, the increased number of osteo- clasts generated in vitro reflects either an increase in sensitivity to $R A N K L$, or an increased number of committed osteoclast precursors in the BMMs isolated from the conditional knockout mice, or both. Further studies will be required to address this question.

The mechanism by which the deletion of Ctsk regulates the intracellular expression of Sphk1 and production of S1P remains to be determined. Secreted cathepsin K, or a factor released by cathepsin $\mathrm{K}$ as it degrades the bone matrix, could act in a paracrine fashion on osteoclasts to regulate Sphk1 expression. Alternatively, evidence is accumulating that cathepsin $\mathrm{K}$ acts intracellularly in addition to its extracellular function of degrading bone matrix proteins. This protease acts within lysosomes to activate TRAP (10), and it is conceivable that a lack of activated TRAP also affects osteoclast biology after Ctsk deletion. Osteoclasts transport protons and chloride ions into the resorbing compartment to dissolve the bone mineral phase and secrete lysosomal acid hydrolases such as cathepsin $\mathrm{K}$ to digest bone matrix proteins. Cathepsin $\mathrm{K}$ is sorted intracellularly into secretory lysosomes, which translocate to and fuse with the ruffled border membrane, releasing their content into the resorption compartment (26). The molecules controlling the trafficking and fusion of lysosomes with the ruffled border membrane therefore play a critical role in bone resorption. Synaptotagmin 7, a $\mathrm{Ca}^{2+}$-sensitive synaptotagmin isoform, regulates exocytosis of lysosomes in osteoclasts, where it colocalizes with LAMP2 and cathepsin K (27). We observed intracellular accumulation of lysosomal vesicles that are positive for synaptotagmin 7 and LAMP2 after Ctsk deletion, similar to what has been reported after pharmacological inhibition of cathepsin K (26). Although it is not possible to determine whether this is the result of impaired vesicular fusion with the plasma membrane, or of increased generation of lysosomes in an attempt to compensate for the defective activity resulting from Ctsk deletion, these changes seem to be specific to Ctsk deletion. In addition, we did not observe an accumulation of secretory lysosomes in Src-deleted osteoclasts (Supplemental Figure 4), which favors the hypothesis of a specific intracellular role of cathepsin $\mathrm{K}$ in affecting both vesicular traffic and S1P synthesis.

From a practical point of view, this study suggests that it should be possible to inhibit bone resorption without impairing bone formation in patients with osteoporosis by inhibiting cathepsin K activity. Indeed, it has recently been reported that inhibition of cathepsin K activity with orally administered small molecules not only inhibits the production of markers of bone resorption, but also maintains or enhances bone formation in some animal models $(32,33,41,42)$, and maintains bone formation markers close to control values in human studies (43, 44). Our mouse genetic studies would suggest that the effect of inhibiting cathepsin $\mathrm{K}$ on bone formation is indirect and is due to the effect of the compounds on osteoclasts rather than on cells of the osteoblast lineage. It is, however, more difficult to understand how such a mechanism of action could explain the reported increase in periosteal bone formation in nonhuman primates (32), since osteoclasts are rarely seen at the periosteum. Our finding that total cortical cross-sectional volume and cortical bone volume were increased after deletion of Ctsk in cells of the osteoclast lineage also implies an increase in periosteal bone formation. Thus, periosteal changes could be secondary to osteoclast-mediated events, which are possibly transduced by the cortical osteocytic network. Alternatively, deletion of Ctsk in monocytes-macrophages residing in the periosteum could be responsible for this increased activity. Further work will be 


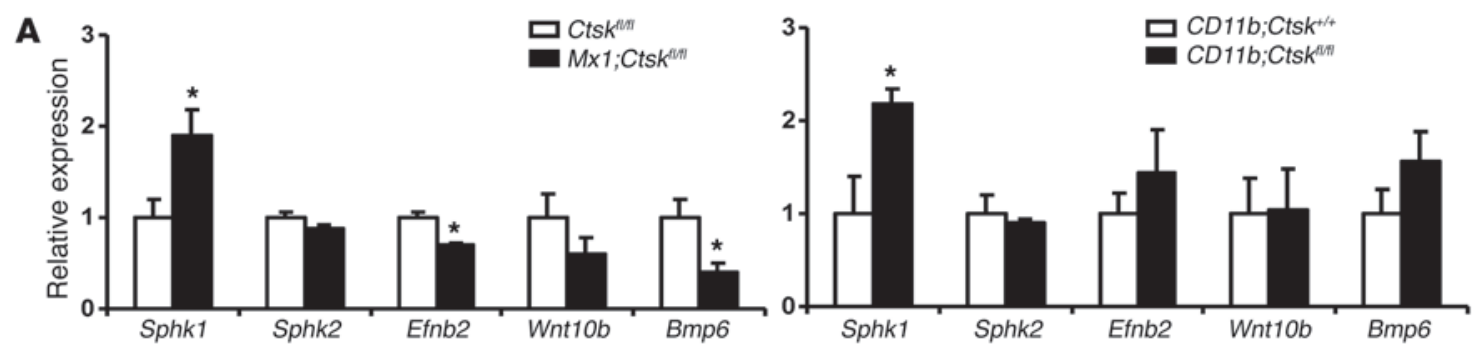

B
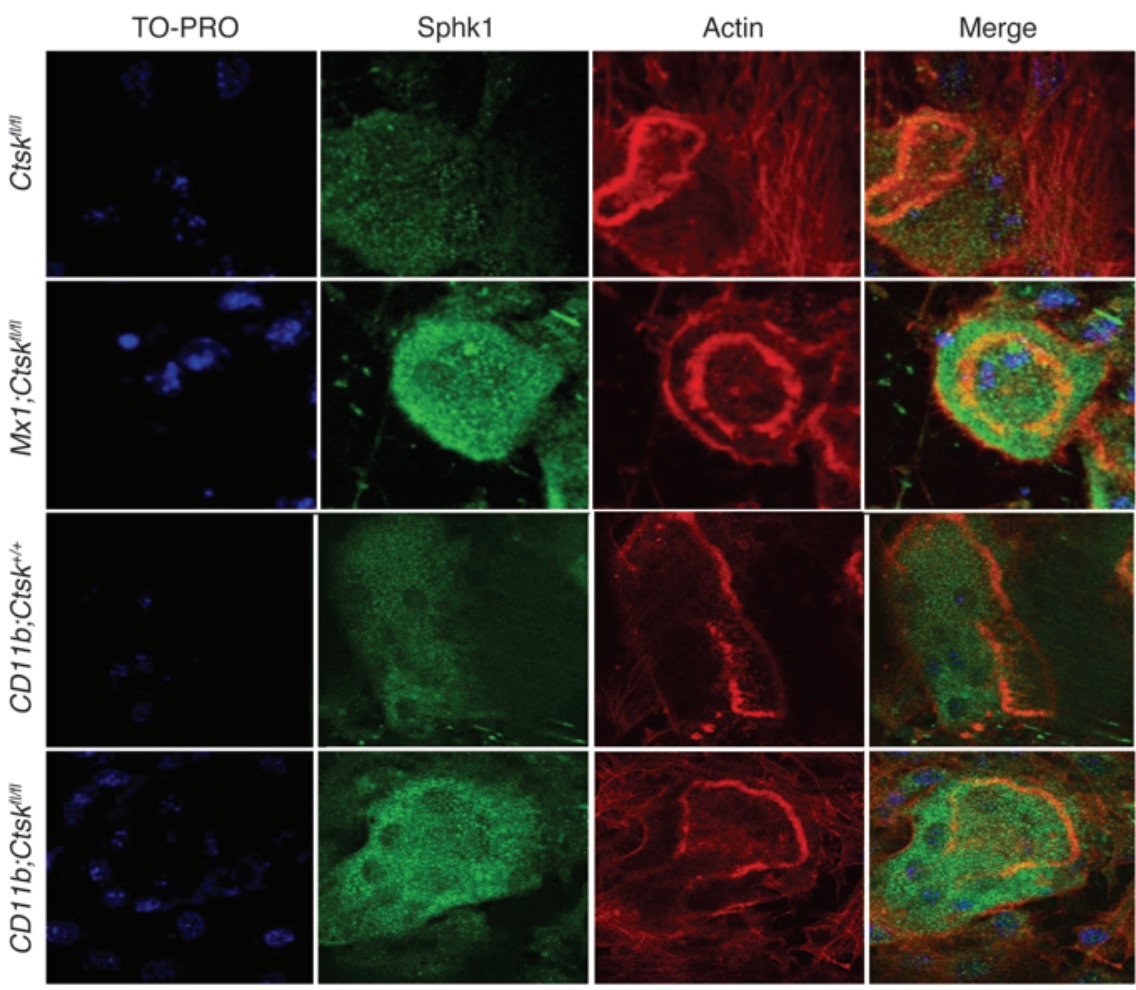

C

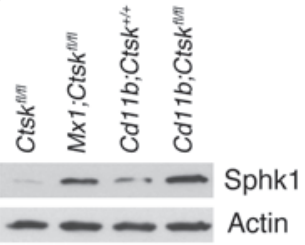

Figure 11

Deletion of Ctsk in hematopoietic cells and osteoclasts increases Sphk1 mRNA expression and protein level in conditional knockout osteoclasts. (A) qRT-PCR analysis of mRNA expression in control and conditional knockout osteoclasts. Data are expressed as percentage of mRNA level of controls. Results are mean \pm SEM. ${ }^{*} P<0.05$ versus controls. (B) Osteoclasts generated by coculture with osteoblasts on collagen gel in the presence of vitamin $\mathrm{D}_{3}$ and $\mathrm{PGE}_{2}$ were replated onto dentin slices and cultured for 48 hours. The localization of TO-PRO-3 (blue), Sphk1 (green), and actin (red) were analyzed by confocal microscopy. Original magnification, $\times 63$. (C) Lysates from osteoclasts were subjected to immunoblotting with anti-Sphk1 antibodies.

required to determine whether this also occurs in humans and to explain this phenomenon.

In conclusion, this study demonstrates that events occurring within osteoclasts have a strong influence on the number of osteoblast progenitors and bone formation in vivo. It also demonstrates in an in vivo setting that the bone resorbing activity of osteoclasts can be dissociated from their ability to stimulate bone formation, such that the coupling process is dependent not only on putative MDGFs, but also on osteoclast-derived molecules - "clastokines"- among which S1P appears to be an important player.

\section{Methods}

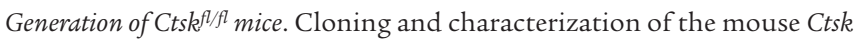
gene have been described previously (45). Plasmids for the neomycin resistance gene (pPGKneobpA-lox B) and for the loxP sequence (pBSloxP) were gifts from Richard Behringer and Reinhard Fässler, respectively. To construct the pG3NEOBLoxP-2 targeting vector, the floxed neomycin resistance gene expression cassette was inserted into intron 5 of the Ctsk gene in reverse orientation as a BamHI-EcoRI fragment (Figure 1A). A third loxP site was inserted into intron 4 . The allele was otherwise left intact. ES cell targeting was performed using standard techniques as previously described (17).

The colonies were screened using Southern analysis. Genomic DNA was digested with BamHI, size-fractioned in 0.8\% agarose gel, and transferred onto Hybond-N+ membrane (GE Healthcare Life Sciences). Membranes were hybridized in a standard buffer at $67^{\circ} \mathrm{C}$ overnight with the pMCK-5 probe that was labeled with $\left[\alpha-{ }^{32} \mathrm{P}\right] \mathrm{dCTP}$ using the random-primed method (Roche Applied Science). The signal was detected using a Fuji BAS 5000 phosphorimager (Fujifilm). The hybridization produced a wild-type band of $11 \mathrm{~kb}$ and a mutant band of $9.5 \mathrm{~kb}$ (Figure 1B). 

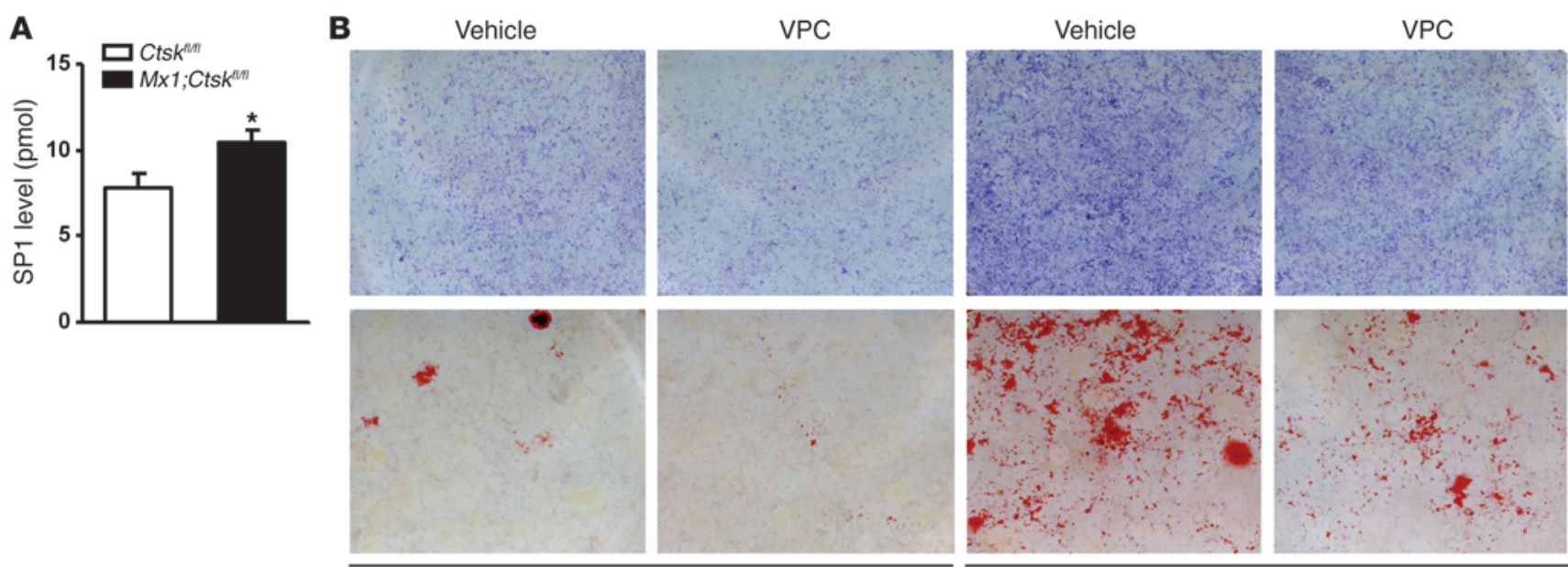

Ctsk $k^{\text {IIn }}$

$M \times 1 ;$ Ctsk $^{\text {int }}$
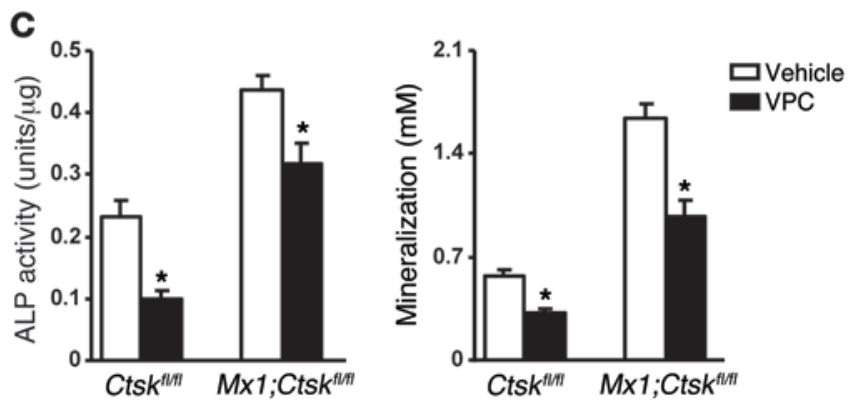

\section{Figure 12}

S1P is increased in the absence of cathepsin K, and blocking S1P activity attenuates ALP activity and mineralization induced by Ctsk-deficient and control osteoclast-conditioned medium. (A) Mx1;Ctsk $k^{f / f l}$ osteoclast-conditioned medium contained elevated levels of S1P. (B) CD1 calvarial osteoblasts were treated with either $M \times 1 ; C t s k^{f l / f l}$ or Ctsk $k^{f l / f l}$ osteoclast-conditioned medium for 7 days and stained with ALP, or for 20 days and stained with alizarin red in the presence or absence of VPC23019 (VPC). Original magnification, $\times 1$. (C) ALP activity ( $n=5$ per group) and mineralization ( $n=6$ per group) were quantified. Results are mean \pm SEM. ${ }^{*} P<0.05$ versus controls.

Northern analysis. For Northern hybridizations, $10-\mu \mathrm{g}$ aliquots of total humerus RNA were denatured with glyoxal, electrophoresed on $0.8 \%$ agarose gels, and blotted onto Biodyne A nylon membranes. The membranes were prehybridized, hybridized, washed, and rehybridized as recommended by the supplier. Northern hybridization for mouse cathepsin $\mathrm{K}$ was performed with the PMCatK-1 cDNA probe (46) labeled by the random-primed method (Roche Applied Science) using $\left[\alpha-{ }^{32} \mathrm{P}\right] \mathrm{dCTP}$. The signals were detected using a Fuji BAS 5000 phosphorimager (Fujifilm), and quantified using TINA 2.0 software (Raytest Isotopenmessgeräte $\mathrm{GmbH}$ ). $p Q C T$. Femoral BMD was measured using the Stratec XCT Research M device with software version 5.40 (Norland Stratec Medizintechnik). For cancellous bone, the scan lines were adjusted to $2.5-4.5 \mathrm{~mm}$ with $0.5-\mathrm{mm}$ intervals at distal metaphysis using the scout view given by the PQCT device. The scan line was adjusted to $7 \mathrm{~mm}$ from the distal end of the femur for cortical bone analysis. All measurements were made using a voxel size of $0.070 \times 0.070 \times 0.5 \mathrm{~mm}^{3}$. Threshold values of $710 \mathrm{mg} / \mathrm{cm}^{3}$ and $350 \mathrm{mg} / \mathrm{cm}^{3}$ were used in the measurements of cortical bone and cancellous bone, respectively.

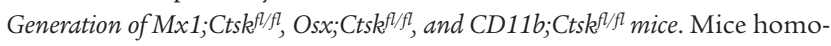
zygous for floxed Ctsk alleles (Ctsklffl) were crossed with those expressing Cre recombinase under the control of a type I interferon-inducible $\mathrm{Mx} 1$ promoter to generate $\mathrm{M} x 1 ; \mathrm{Ctsk} \mathrm{fl}^{l /+}$ mice. $\mathrm{M} x 1 ; \mathrm{Ctsk}^{\mathrm{ll} /+}$ mice were crossed with

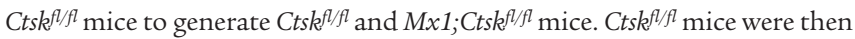
crossed with $M x 1 ; C t s k l / f l$ mice to obtain approximately $50 \%$ conditional knockouts and controls. We compared the skeletal phenotype of Cre-negative and Cre-positive mice, as other investigators have done $(21,25,47)$. Activation of Mx1-Cre was accomplished by i.p. injection of 3 doses of 10 $\mu \mathrm{g} / \mathrm{g}$ body weight polyinosinic-polycytidylic acid (polyI:C) every other day at 2 weeks of age. Similar to $M x 1 ; C_{t s k} l^{l / f l}$ mice, $C t s k^{f / / l}$ mice were crossed with mice expressing Cre under the control of osterix promoter to generate $O s x$; $\mathrm{Ctsk}^{f /+}$ mice. Since Osx-Cre mice are mildly osteopenic (28), we crossed Osx; $\mathrm{Ctsk}^{f /+}$ mice with $\mathrm{Ctsk}^{l /+}$ mice to generate $\mathrm{Osx} ; \mathrm{Ctsk}^{+/+}$and $O s x ; \mathrm{Ctsk}^{f / / f l}$ mice. $\mathrm{CD} 11 b$-Cre is on the Y chromosome. Thus, all males are $\mathrm{Cre}$-positive and the only Cre-negative mice are females. Male CD11b; $\mathrm{Ctsk}^{\mathrm{fl} /+}$ mice were crossed with female Ctsk $\mathrm{fl}^{l /+}$ mice to generate $C D 11 b ; \mathrm{Ctsk}^{+/+}$and CD11b;Ctsk fl/fl mice. The skeletal phenotype of male conditional knockouts and controls was compared due to sex differences in bone volume.

PCR genotype analysis. Genomic DNA isolated from tail biopsies was used to genotype mice. The Cre transgene was detected by PCR using the forward primer (5'-CGCGGTCTGGCAGTAAAAACTATC-3'), and reverse primer (5'-CCCACCGTCAGTACGTGAGATATC-3'). The floxed and wildtype alleles were determined using 3 primers: P1 (5'-GGAAACTGAGGCACAGAGTTCAG-3'); P2 (5'-CTGTCTTTGTTGTGAAGCCCTGCT-3'); and P3 (5'-GGGGTGTGCACCTATGTGTGTA-3'). The deleted floxed allele was identified using primers $\mathrm{P} 1$ and $\mathrm{P} 3$ for $M x 1 ; \mathrm{Ctsk}^{f / f l}$ and their controls, and primers P1-3 for Osx; $\mathrm{Ctsk}^{\mathrm{fl} / \mathrm{fl}}, \mathrm{CD} 11 \mathrm{~b} ; \mathrm{Ctsk} \mathrm{fl}^{l / f}$, and respective controls.

Histomorphometry. Nine-week-old Ctskfl/fl, Mx1;Ctskfl/fl, CD11b;Ctsk ${ }^{+/+}$, and CD11b;Ctskl/fl mice were subcutaneously injected with $20 \mathrm{mg} / \mathrm{kg}$ cal- 
A

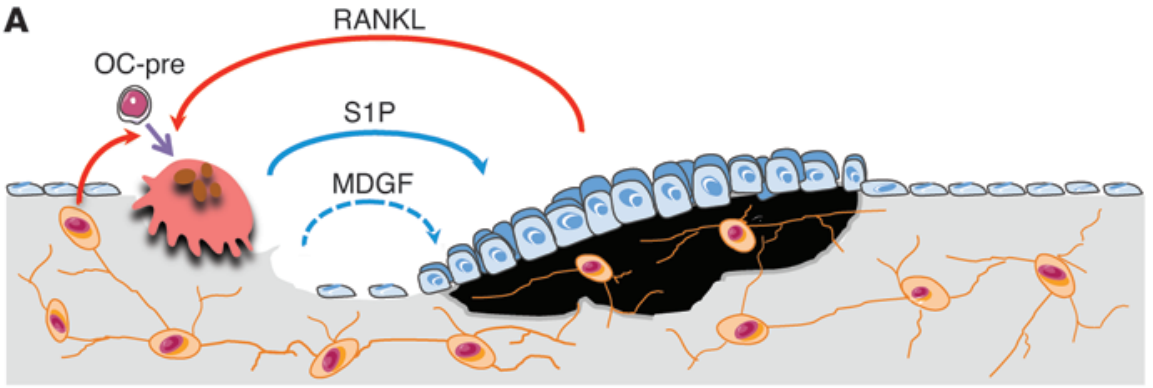

B

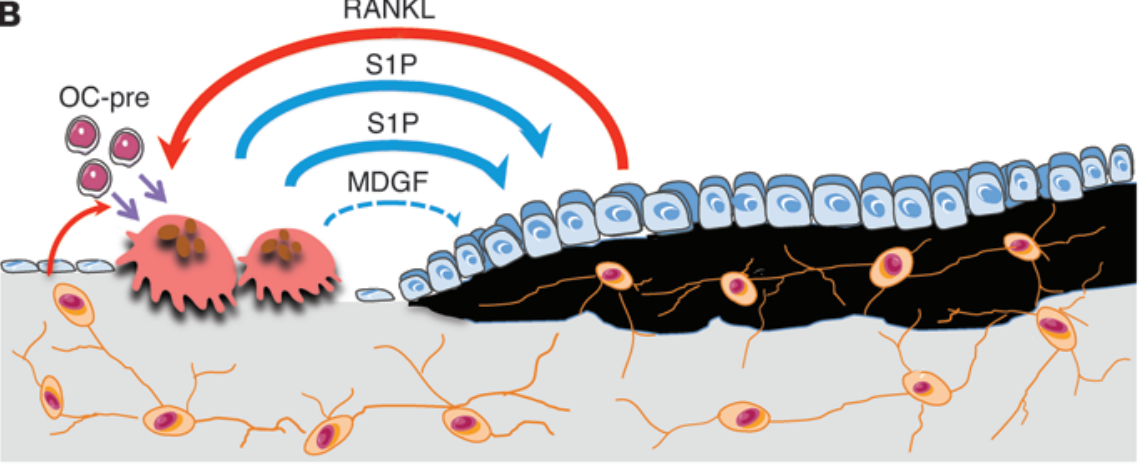

Figure 13

Schematic representation of bone remodeling and the coupling process in controls and Ctsk-deficient mice. (A) Under normal circumstance, osteoclasts secrete clastokines, including osteoanabolic factors such as S1P, for the regulation of bone formation. Additionally, osteoclasts resorb bone, thus releasing MDGFs to enhance bone formation. (B) Absence of cathepsin $\mathrm{K}$ increases S1P released by osteoclasts and may increase intact MDGFs (11). Furthermore, S1P induces an increase in the production of RANKL by osteoblasts (20), leading to increased osteoclast differentiation. An overall increase in the level of S1P stimulates bone formation. OC-pre, osteoclast precursor. cein (Sigma-Aldrich) and demeclocycline (Sigma-Aldrich) on days 7 and 2 before necropsy, respectively. At the end of the experiment, the mice were weighed and anesthetized with isoflurane. To analyze the effect of targeted deletion of Ctsk in the osteoblast lineage, 13-week-old Osx-Cre; Ctsk ${ }^{+/+}$and $\mathrm{Osx}$-Cre; $\mathrm{Ctsk}^{\mathrm{fl} / \mathrm{fl}}$ mice were fluorochrome injected on days 9 and 2 before necropsy. Mice were killed by cardiectomy. Femurs were removed and fixed in $70 \%$ alcohol. They were dehydrated in ethanol, infiltrated, and embedded without demineralization in methyl methacrylate. Longitudinal $5-\mu \mathrm{m}$ thick sections were cut on a Reichert-Jung Supercut 2165 microtome (Leica) and mounted unstained for static and dynamic measurements. A standard sampling site with an area of approximately $2.5 \mathrm{~mm}^{2}$ was established in the secondary spongiosa of the metaphysis. Histomorphometric measurements were carried out semiautomatically with the OsteoMeasure image analyzer (Osteometrics Inc.). All parameters were expressed according to standardized nomenclature (48). Bone volume per tissue volume (BV/TV, \%), trabecular thickness (Tb.Th, $\mu \mathrm{m})$, trabecular separation (Tb.Sp, $\mu \mathrm{m})$, trabecular number (Tb.N, $/ \mathrm{mm}$ ), and mineralizing surface per bone surface (MS/BS, \%) were measured. MAR was defined as the mean distance between the fluorescent labels divided by the interlabeling period. BFR was calculated as the product of MS/BS and MAR, and expressed per bone surface (BFR/BS, $\mu \mathrm{m}^{3} / \mu \mathrm{m}^{2} /$ year), bone volume (BFR/BV, \%/year), and tissue volume referent (BFR/TV, \%/year). Osteoblast number per tissue area (N.Ob/T.Ar, $/ \mathrm{mm}^{2}$ ) was determined by identifying large basophilic cuboidal cells in toluidine blue-stained sections. Osteoclast number per tissue area (N.Oc/T.Ar, $/ \mathrm{mm}^{3}$ ) was determined by identifying multinucleated cells.

$\mu C T$. High-resolution images of the femur were acquired using a desktop $\mu \mathrm{CT} 35$ (Scanco Medical), according to the recommended guidelines (49). Trabecular bone microarchitecture in the distal femoral metaphysis and cortical bone at the femoral midshaft were determined using a $7-\mu \mathrm{m}$ isotropic voxel size, $50 \mathrm{kVp}$, and $144 \mu \mathrm{A}$. Cancellous bone was assessed in the region starting $120 \mu \mathrm{m}$ proximal to the growth plate and extending 464 transverse slices. Scans were subjected to Gaussian filtration and segmentation using a fixed threshold at $26 \%$ of maximal gray scale value. Bone volume fraction (BV/TV, -); trabecular number (Tb.N, /mm); trabecular thickness (Tb.Th, mm); trabecular separation (Tb.Sp, mm); connectivity density (ConnD, $/ \mathrm{mm}^{3}$ ); and structural model index (SMI, -) were analyzed. For cortical bone, 86 transverse $\mu \mathrm{CT}$ slices were evaluated using a threshold at $35 \%$ of maximal gray scale value. Total cross-sectional volume $\left(\mathrm{mm}^{3}\right)$, cortical volume $\left(\mathrm{mm}^{3}\right)$, marrow volume $\left(\mathrm{mm}^{3}\right)$, and cortical thickness $(\mathrm{mm})$ were assessed.

Preparation of osteoblasts from long bones. A mature osteoblast-enriched bone cell population was obtained from tibiae and femurs and isolated using a modified method (50). In brief, bones were cleaned of adhering tissues, minced, and digested in serum-free $\alpha$-MEM (Invitrogen; Life Technologies) containing $100 \mathrm{units} / \mathrm{ml}$ penicillin, $100 \mu \mathrm{g} / \mathrm{ml}$ streptomycin, and collagenase type II (Worthington Biochemical) for 2 hours at $37^{\circ} \mathrm{C}$. The bone fragments were transferred to $75-\mathrm{cm}^{2}$ flasks containing $\alpha$-MEM, $10 \%$ FBS, 100 units $/ \mathrm{ml}$ penicillin, and $100 \mu \mathrm{g} / \mathrm{ml}$ streptomycin. Medium was replaced every 2 days until cells were confluent.

$A L P$ and mineralization assay. Osteoblasts derived from long bones were plated at $1 \times 10^{4}$ cells per well in 12 -well plates. Three days after plating, the culture medium was replaced with a differentiation medium containing $\alpha$-MEM supplemented with $10 \%$ FBS, 100 units/ml penicillin, $100 \mu \mathrm{g} /$ $\mathrm{ml}$ streptomycin, $5 \mathrm{mM} \beta$-glycerophosphate, $10 \mu \mathrm{M}$ dexamethasone, and $50 \mu \mathrm{g} / \mathrm{ml}$ ascorbic acid. Medium was replaced every 3 days. On days 7 and 20 , cells were fixed with $3.7 \%$ formaldehyde and stained for ALP activity or mineralized matrix with Fast Blue Alkaline Phosphatase (Sigma-Aldrich) and $2 \%$ alizarin red (Sigma-Aldrich) for 45 minutes and 20 minutes, respectively. For conditioned medium experiments, CD1 calvarial osteoblasts were plated at $2 \times 10^{4}$ and $1 \times 10^{4}$ cells per well in 12-well plates for ALP and mineralization assays, respectively. VPC23019 (Avanti Polar Lipids) at 2 and $10 \mu \mathrm{M}$ was added to the medium. ALP activity was determined according to the manufacturer's protocol (LabAssay ALP; Wako Chemicals USA). Mineralized bone nodules stained with alizarin red were destained with $10 \%$ cetylpyridinium chloride in $10 \mathrm{mM}$ sodium phosphate ( $\mathrm{pH} 7$ ), and the mineralization was quantified (51).

Preparation of osteoclasts. Osteoclasts were generated on dentin slices, tissue culture dishes, or coverslips. BM cells were cultured in $\alpha$-MEM containing 
$10 \%$ FBS, 100 units $/ \mathrm{ml}$ penicillin, and $100 \mu \mathrm{g} / \mathrm{ml}$ streptomycin for 24 hours to generate BMMs. For the in vitro resorption assay, BMMs were cultured with primary calvarial osteoblasts obtained from CD1 mice in $\alpha$-MEM containing $10 \mathrm{nM} 1,25$-dihydroxyvitamin $\mathrm{D}_{3}$ and $1 \mu \mathrm{M}$ prostaglandin $\mathrm{E}_{2}$ on a collagen gel (52). Collagen was digested with $0.1 \%$ collagenase, and osteoclasts were replated onto dentin slices for an additional 48 hours to determine bone-resorbing activity. To produce osteoclast-conditioned medium and for immunofluorescence and TRAP staining, BMMs were cultured on tissue culture plastic or coverslips in $\alpha$-MEM for 2 days with M-CSF (20 ng/ $\mathrm{ml}$ ), and for an additional 6 days in the same medium with M-CSF (20 ng/ $\mathrm{ml})$ and RANKL $(3.3 \mathrm{ng} / \mathrm{ml})$ to avoid contamination with osteoblasts.

Confocal microscopy. Osteoclasts plated on dentin slices were fixed with 3.7\% formaldehyde in PBS for 10 minutes. Cells were permeabilized in $0.05 \%$ saponin for 30 minutes followed by acetone for 1 minute. Coverslips for actin labeling were extracted in ice-cold acetone for 3-5 minutes, incubated in a 1:40 dilution of rhodamine phalloidin stock solution (Invitrogen; Life Technologies) for 1 hour, washed with PBS, and mounted in FluorSave (Calbiochem; EMD Millipore). All coverslips were blocked in 5\% normal goat serum (Roche Applied Science) for 30 minutes and incubated in appropriate primary antibodies (SPHK1, LAMP2, and synaptotagmin 7), washed, incubated with fluorescent secondary antibody (Alexa Fluor 488, green; 568, red; and 647, blue), washed again, and mounted in FluorSave (Calbiochem; EMD Millipore). Nuclei were labeled with TO-PRO-3 (1:1000) in the secondary antibody solution. Fixed cells were visualized using a 510 Meta laser scanning confocal microscope (Carl Zeiss), and images were recorded.

Bone resorption assay. Following culture with osteoclasts, dentin slices were washed with PBS and sonicated in $1 \mathrm{~N} \mathrm{NaOH}$ for 1 minute to remove cells. Resorption pit images were captured using a 510 Meta laser scanning confocal microscope (Carl Zeiss) and analyzed with Fuji software.

qRT-PCR. Total RNA was extracted from femurs, cultured osteoblasts derived from long bones, and cultured osteoclasts using a monophasic solution of guanidine isothiocyanate and phenol according to the manufacturer's instructions (TRIzol Reagent; Invitrogen; Life Technologies) followed by RNA clean-up using RNeasy Mini Kit (QIAGEN). The RNA yields were determined spectrophotometrically at $260 \mathrm{~nm}$. First-strand cDNA was synthesized using $1 \mu \mathrm{g}$ of total RNA and SuperScript II (Invitrogen; Life Technologies) for reverse transcription PCR. The qRT-PCR was performed at $57^{\circ} \mathrm{C}$ for 40 cycles using an iCycler (Bio-Rad Laboratories). The samples were normalized to GAPDH expression. Primer sequences are presented in Supplemental Table 7.

FACS analysis. Osteoblasts derived from long bones were incubated with PE-conjugated anti-alkaline phosphatase antibody (R\&D Systems) for 30 minutes at $4^{\circ} \mathrm{C}$. Cells were washed twice with washing buffer. Stained cells were suspended in PBS and flow cytometry was performed using LRSFortessa (BD Biosciences).

$\mathrm{CFU}$ assays. Osteoblasts derived from long bones of $M x 1 ; \mathrm{Ctsk}^{l / f l}$ mice and their control littermates were cultured in $\alpha$-MEM supplemented with $10 \%$ FBS, $100 \mathrm{unit} / \mathrm{ml}$ penicillin, $100 \mu \mathrm{g} / \mathrm{ml}$ streptomycin, $5 \mathrm{mM} \beta$-glycerophosphate, $10 \mu \mathrm{M}$ dexamethasone, and $50 \mu \mathrm{g} / \mathrm{ml}$ ascorbic acid. CFU-ALP was determined by counting ALP-positive colonies on day 7. For the determination of CFU-OBs, cells were maintained in differentiation medium for 20 days, and mineralized nodules were stained using alizarin red. The total number of colonies or CFU-Fs was determined by toluidine blue staining on days 7 and 20 in the same tissue culture plate.

S1P lipid extraction and analysis. Five million nonadherent BM cells were plated onto 6-well plates in the presence of M-CSF and RANKL to generate osteoclasts. On day 5, cells were rinsed with PBS 3 times and grown in $\alpha$-MEM without serum for 9 hours. Conditioned medium was collected, centrifuged, and kept at $-80^{\circ} \mathrm{C}$ until processed for mass spectrometry.
S1P was extracted by a modified two-step lipid extraction technique previously described $(53,54)$. The medium $(1 \mathrm{ml})$ was transferred into a siliconized glass tube and 100 pmol C17-S1P was added as internal standard. After alkalization with $100 \mu \mathrm{l}$ of a $3 \mathrm{~N} \mathrm{NaOH}$ solution, lipids were extracted by addition of $1 \mathrm{ml}$ chloroform and $1 \mathrm{ml}$ methanol/ $\mathrm{HCl}(99.8: 0.2 \mathrm{v} / \mathrm{v})$. The mixture was centrifuged ( $300 \mathrm{~g}$ for 5 minutes) to separate the alkaline aqueous phase containing S1P. The organic phase was re-extracted with $0.5 \mathrm{ml}$ methanol, $0.5 \mathrm{ml} 1 \mathrm{~N} \mathrm{NaCl}$, and $50 \mu \mathrm{l} 3 \mathrm{~N} \mathrm{NaOH}$. The collected aqueous phases were acidified with $100 \mu \mathrm{l}$ concentrated $\mathrm{HCl}$ and extracted twice with $1.5 \mathrm{ml}$ chloroform each. The combined organic phases were evaporated using a Speed-Vac SC201 ARC vacuum system (Thermo Fisher Scientific), and the dried lipids were redissolved in $200 \mu \mathrm{l}$ methanol by rigorous vortexing and sonification on ice.

Sample analysis was performed using rapid-resolution liquid chromatography tandem mass spectrometry (LC-MS/MS). Chromatographic separations were obtained using a Waters X-Bridge $\mathrm{C} 18$ separation column $(4.6 \mathrm{~mm} \times 150 \mathrm{~mm}, 3.5 \mu \mathrm{m}$ particle size, $138 \AA$ pore size) with a Waters X-Bridge $\mathrm{C} 18$ guard column $(4.6 \times 20 \mathrm{~mm}$; Waters). We chose a binary solvent system with gradient elution consisting of eluent $\mathrm{A}$ (water/formic acid; 100:0.1 v/v); and eluent B (acetonitril/tetrahydrofuran/formic acid; 50:50:0.1 v/v); and a flow rate of $0.5 \mathrm{ml} / \mathrm{min}$ over 15 minutes. Mass spectrometric measurements were conduct using the following ion source conditions and gas settings for positive LC-MS/ MS: sheath gas temperature $=300^{\circ} \mathrm{C}$; sheath gas flow $=91 / \mathrm{min}$; nebulizer pressure $=30$ psig; drying gas temperature $=300^{\circ} \mathrm{C}$; drying gas flow $=8 \mathrm{l} / \mathrm{min}$; capillary voltage $=5400 \mathrm{~V}$; fragmentor voltage $=200 \mathrm{~V}$; nozzle voltage $=2000 \mathrm{~V}$; and collision energy $=15 \mathrm{~V}$. The precursor ions of S1P $(m / z 380.2560)$ and C17 S1P $(m / z$ 366.2404) were cleaved into the fragment ions of $m / z 264.2700$ and $m / z 250.2529$, respectively. Quantification was performed using MassHunter Software (Agilent Technologies). A calibration curve of analyte concentration was performed from 1-150 pmol and constructed by linear fitting using the least squares linear regression calculation. The resulting slope of the calibration curve was then used to calculate the concentration of the respective analyte in the unknowns.

Statistics. Data are expressed as mean \pm SEM in figures or mean \pm SD in tables. An unpaired $t$-test was used to compare 2 group means. Multiple comparisons were analyzed by 1-way ANOVA followed by Fisher's protected least significant difference test. Measurements of $P$ less than 0.05 were considered statistically significant.

Study approval. All mouse experiments were performed in accordance with a protocol approved by the Institutional Animal Care and Use Committee at Harvard Medical School, and followed the U.S. Public Health Service Policy on Humane Care and Use of Laboratory Animals.

\section{Acknowledgments}

We thank Kei Yamana for providing PCR primer sequences. We also thank Nicholas Brady, Chantel Lester, and Antonios Aliprantis for $\mu C T$ analysis. Confocal analysis was performed at the Harvard NeuroDiscovery Center (Boston, Massachusetts, USA). This work was supported in part by Procter \& Gamble.

Received for publication May 16, 2012, and accepted in revised form November 15, 2012.

Address correspondence to: Roland Baron, Department of Oral Medicine, Infection and Immunity, Harvard School of Dental Medicine, 188 Longwood Avenue, REB 310, Boston, Massachusetts 02115, USA. Phone: 617.432.7320; Fax: 617.432.1897; E-mail: roland_baron@hsdm.harvard.edu. 
1. Nyman JS, Yeh OC, Hazelwood SJ, Martin RB. A theoretical analysis of long-term bisphosphonate effects on trabecular bone volume and microdamage. Bone. 2004;35(1):296-305.

2. Bone HG, Schurr W. Intravenous bisphosphonate therapy for osteoporosis: where do we stand? Curr Osteoporos Rep. 2004;2(1):24-30.

3. Khan AA, et al. Bisphosphonate associated osteonecrosis of the jaw. J Rheumatol. 2009;36(3):478-490.

4. Koh JS, Goh SK, Png MA, Kwek EB, Howe TS. Femoral cortical stress lesions in long-term bisphosphonate therapy: a herald of impending fracture? J Orthop Trauma. 2010;24(2):75-81.

5. Carvalho NN, Voss LA, Almeida MO, Salgado CL, Bandeira F. Atypical femoral fractures during prolonged use of bisphosphonates: short-term responses to strontium ranelate and teriparatide. J Clin Endocrinol Metab. 2011;96(9):2675-2680.

6. Black DM, et al. The effects of parathyroid hormone and alendronate alone or in combination in postmenopausal osteoporosis. N Engl J Med. 2003; 349(13):1207-1215.

7. Dougall WC, et al. RANK is essential for osteoclast and lymph node development. Genes Dev. 1999;13(18):2412-2424.

8. Douni E, et al. A RANKL G278R mutation causing osteopetrosis identifies a functional amino acid essential for trimer assembly in RANKL and TNF. Hum Mol Genet. 2012;21(4):784-798.

9. Schinke T, et al. Impaired gastric acidification negatively affects calcium homeostasis and bone mass. Nat Med. 2009;15(6):674-681.

10. Zenger $S$, et al. Proteolytic processing and polarized secretion of tartrate-resistant acid phosphatase is altered in a subpopulation of metaphyseal osteoclasts in cathepsin K-deficient mice. Bone. 2007; 41(5):820-832.

11. Fuller K, et al. Cathepsin $\mathrm{K}$ inhibitors prevent matrix-derived growth factor degradation by human osteoclasts. Bone. 2008;42(1):200-211.

12. Gelb BD, Shi GP, Chapman HA, Desnick RJ. Pycnodysostosis, a lysosomal disease caused by cathepsin K deficiency. Science. 1996;273(5279):1236-1238.

13. Gelb BD, et al. Pycnodysostosis: refined linkage and radiation hybrid analyses reduce the critical region to $2 \mathrm{cM}$ at $1 \mathrm{q} 21$ and map two candidate genes. Hum Genet. 1996;98(2):141-144.

14. Johnson MR, Polymeropoulos MH, Vos HL, Ortiz de Luna RI, Francomano CA. A nonsense mutation in the cathepsin $\mathrm{K}$ gene observed in a family with pycnodysostosis. Genome Res. 1996;6(11):1050-1055.

15. Saftig P, et al. Impaired osteoclastic bone resorption leads to osteopetrosis in cathepsin-K-deficient mice. Proc Natl Acad Sci U S A. 1998;95(23):13453-13458.

16. Gowen M, et al. Cathepsin K knockout mice develop osteopetrosis due to a deficit in matrix degradation but not demineralization. J Bone Miner Res. 1999;14(10):1654-1663.

17. Kiviranta R, et al. Impaired bone resorption in cathepsin $\mathrm{K}$-deficient mice is partially compensated for by enhanced osteoclastogenesis and increased expression of other proteases via an increased RANKL/OPG ratio. Bone. 2005;36(1):159-172.

18. Pennypacker $B$, et al. Bone density, strength, and formation in adult cathepsin $\mathrm{K}(-/-)$ mice. Bone. 2009; 44(2):199-207.

19. Li CY, et al. Mice lacking cathepsin K maintain bone remodeling but develop bone fragility despite high bone mass. J Bone Miner Res. 2006;21(6):865-875.

20. Ryu J, Kim HJ, Chang EJ, Huang H, Banno Y, Kim $\mathrm{HH}$. Sphingosine 1-phosphate as a regulator of osteoclast differentiation and osteoclast-osteoblast coupling. EMBO J. 2006;25(24):5840-5851.

21. Aliprantis AO, et al. NFATc1 in mice represses osteoprotegerin during osteoclastogenesis and dissociates systemic osteopenia from inflammation in cherubism. J Clin Invest. 2008;118(11):3775-3789.

22. Beller DI, Springer TA, Schreiber RD. Anti-Mac-1 selectively inhibits the mouse and human type three complement receptor. J Exp Med. 1982; 156(4):1000-1009.

23. Shalhoub V, et al. Characterization of osteoclast precursors in human blood. Br J Haematol. 2000; 111(2):501-512.

24. Ferron M, Vacher J. Targeted expression of Cre recombinase in macrophages and osteoclasts in transgenic mice. Genesis. 2005;41(3):138-145.

25. Ambrogini E, et al. FoxO-mediated defense against oxidative stress in osteoblasts is indispensable for skeletal homeostasis in mice. Cell Metab. 2010; 11(2):136-146.

26. Leung P, Pickarski M, Zhuo Y, Masarachia PJ, Duong LT. The effects of the cathepsin K inhibitor odanacatib on osteoclastic bone resorption and vesicular trafficking. Bone. 2011;49(4):623-635.

27. Zhao H, Ito Y, Chappel J, Andrews NW, Teitelbaum SL, Ross FP. Synaptotagmin VII regulates bone remodeling by modulating osteoclast and osteoblast secretion. Dev Cell. 2008;14(6):914-925.

28. Davey RA, et al. Decreased body weight in young Osterix-Cre transgenic mice results in delayed cortical bone expansion and accrual. Transgenic Res. 2012;21(4):885-893.

29. Pederson L, Ruan M, Westendorf JJ, Khosla S, Oursler MJ. Regulation of bone formation by osteoclasts involves Wnt/BMP signaling and the chemokine sphingosine-1-phosphate. Proc Natl Acad Sci U S A. 2008;105(52):20764-20769.

30. Zhao C, et al. Bidirectional ephrinB2-EphB4 signaling controls bone homeostasis. Cell Metab. 2006; 4(2):111-121.

31. Irie $\mathrm{N}$, et al. Bidirectional signaling through ephrinA2-EphA2 enhances osteoclastogenesis and suppresses osteoblastogenesis. J Biol Chem. 2009; 284(21):14637-14644.

32. Cusick T, et al. Odanacatib treatment increases hip bone mass and cortical thickness by preserving endocortical bone formation and stimulating periosteal bone formation in the ovariectomized adult rhesus monkey. J Bone Miner Res. 2012;27(3):524-537.

33. Jerome C, Missbach M, Gamse R. Balicatib, a cathep$\sin \mathrm{K}$ inhibitor, stimulates periosteal bone formation in monkeys. Osteoporos Int. 2012;23(1):339-349.

34. Silva BC, Costa AG, Cusano NE, Kousteni S, Bilezikian JP. Catabolic and anabolic actions of parathyroid hormone on the skeleton. J Endocrinol Invest. 2011;34(10):801-810.

35. Ilmer M, Karow M, Geissler C, Jochum M, Neth P. Human osteoblast-derived factors induce early osteogenic markers in human mesenchymal stem cells. Tissue Eng Part A. 2009;15(9):2397-2409.

36. Podgorski I, et al. Bone marrow-derived cathepsin K cleaves SPARC in bone metastasis. Am J Pathol. 2009; 175(3):1255-1269.

37. Mandelin J, et al. Human osteoblasts produce cathepsin K. Bone. 2006;38(6):769-777.
38. Tang Y, et al. TGF- $\beta 1$-induced migration of bone mesenchymal stem cells couples bone resorption with formation. Nat Med. 2009;15(7):757-765.

39. Xiong J, Onal M, Jilka RL, Weinstein RS, Manolagas SC, O'Brien CA. Matrix-embedded cells control osteoclast formation. Nat Med. 2011;17(10):1235-1241.

40. Nakashima T, et al. Evidence for osteocyte regulation of bone homeostasis through RANKL expression. Nat Med. 2011;17(10):1231-1234.

41. Masarachia PJ, et al. Odanacatib reduces bone turnover and increases bone mass in the lumbar spine of skeletally mature ovariectomized rhesus monkeys. J Bone Miner Res. 2012;27(3):509-523.

42. Pennypacker BL, et al. Cathepsin K inhibitors prevent bone loss in estrogen-deficient rabbits.J Bone Miner Res. 2011;26(2):252-262.

43. Vasiljeva O, Reinheckel T, Peters C, Turk D, Turk $\mathrm{V}$, Turk B. Emerging roles of cysteine cathepsins in disease and their potential as drug targets. Curr Pharm Des. 2007;13(4):387-403.

44. Costa AG, Cusano NE, Silva BC, Cremers S, Bilezikian JP. Cathepsin K: its skeletal actions and role as a therapeutic target in osteoporosis. Nat Rev Rheumatol. 2011;7(8):447-456.

45. Rantakokko J, Kiviranta R, Eerola R, Aro HT, Vuorio E. Complete genomic structure of the mouse cathepsin K gene (Ctsk) and its localization next to the Arnt gene on mouse chromosome 3. Matrix Biol. 1999;18(2):155-161.

46. Rantakokko J, Aro HT, Savontaus M, Vuorio E. Mouse cathepsin K: cDNA cloning and predominant expression of the gene in osteoclasts, and in some hypertrophying chondrocytes during mouse development. FEBS Lett. 1996;393(2-3):307-313.

47. Ruocco MG, et al. I\{kappa\}B kinase (IKK) \{beta\}, but not IKK $\{$ alpha\}, is a critical mediator of osteoclast survival and is required for inflammation-induced bone loss. J Exp Med. 2005;201(10):1677-1687.

48. Parfitt AM, et al. Bone histomorphometry: standardization of nomenclature, symbols, and units. Report of the ASBMR Histomorphometry Nomenclature Committee. J Bone Miner Res. 1987;2:595-610.

49. Bouxsein ML, Boyd SK, Christiansen BA, Guldberg RE, Jepsen KJ, Muller R. Guidelines for assessment of bone microstructure in rodents using microcomputed tomography. J Bone Miner Res. 2010; 25(7):1468-1486.

50. Bakker AD, Klein-Nulend J. Osteoblast isolation from murine calvaria and long bones. Methods Mol Biol. 2012;816:19-29.

51. Lotinun S, Evans GL, Turner RT, Oursler MJ. Deletion of membrane-bound steel factor results in osteopenia in mice. J Bone Miner Res. 2005;20(4):644-652.

52. Purev E, Neff L, Horne WC, Baron R. c-Cbl and Cbl-b act redundantly to protect osteoclasts from apoptosis and to displace HDAC6 from beta-tubulin, stabilizing microtubules and podosomes. Mol Biol Cell. 2009;20(18):4021-4030.

53. Ruwisch L, Schafer-Korting M, Kleuser B. An improved high-performance liquid chromatographic method for the determination of sphingosine-1phosphate in complex biological materials. Naunyn Schmiedebergs Arch Pharmacol. 2001;363(3):358-363.

54. Yatomi Y, Ruan F, Ohta J, Welch RJ, Hakomori S, Igarashi Y. Quantitative measurement of sphingosine 1-phosphate in biological samples by acylation with radioactive acetic anhydride. Anal Biochem. 1995;230(2):315-320. 